\title{
Distributions in algebraic dynamics
}

\author{
Shou-Wu Zhang
}

\section{Contents}

$\begin{array}{ll}0 . & \text { Introduction } \\ \end{array}$

1. Kähler and algebraic dynamics 383

2. Classifications 394

3. Canonical metrics and measures 403

4. Arithmetic dynamics 416

$\begin{array}{ll}\text { References } & 428\end{array}$

\section{Introduction}

The complex dynamic system is a subject to study iterations on $\mathbb{P}^{1}$ or $\mathbb{P}^{N}$ with respect to complex topology. It originated from the study of Newton method and the three body problem in the end of 19th century and was highly developed in 20th century. It is a unique visualized subject in pure math because of the beautiful and intricate pictures of the Julia sets generated by computer. The subject of this paper, algebraic dynamics, is a subject to study iterations under Zariski topology and is still in its infancy. If the iteration is defined over a number field, then we are in the situation of arithmetical dynamics where the Galois group and heights will be involved. Here we know very little besides very symmetric objects like abelian varieties and multiplicative groups.

The development of arithmetical dynamics was initiated by Northcott in his study of heights on projective space [47], 1950. He showed that the set of rational preperiodic points of any endomorphism of $\mathbb{P}^{N}$ of degree $\geq 2$ is always finite. The modern theory of canonical heights was developed by Call and Silverman in [11]. Their theory generalized earlier notions of Weil heights on projective spaces and Néron-Tate heights on abelian varieties. Thus many classical questions about abelian varieties and multiplicative groups can be asked again for the dynamical system, such as the size of 
rational points, preperiodic points, and their distributions. See $\S 4.1 .5$ and $\S 4.1 .6$ for some standard conjectures, such as Lehmer's conjecture and Lang's conjecture. In [62], we developed a height theory for subvarieties and an intersection theory for integrable adelic metrized line bundles, based on Gillet and Soulé's intersection theory [26]. Thus many questions about points can be asked again for subvarieties. Two questions we considered in [62] are the Manin-Mumford conjecture and the Bogomolov conjecture. See Conjecture 1.2.1 and 4.1.1.

This note in a large sense is an extension of our previous paper. Our first goal is to provide a broad background in Kähler geometry, algebraic geometry, and measure theory. Our second goal is to survey and explain the new developments. The following is a detailed description of the contents of the paper.

In $\S 1$, we will give some basic definitions and examples of dynamics in Kähler geometry and algebraic geometry, and study the Zariski properties of preperiodic points. Our dynamic Manin-Mumford conjecture says that a subvariety is preperiodic if and only if it contains many preperiodic points. One question we wish to know (but don't yet) is about the positivity of a canonical $(1,1)$ class on the moduli of cycles on a Kähler variety.

In $\S 2$, we will study the classification problem about Kähler dynamics. In surfaces, the problem can be completely solved. In the smooth projective case, we will prove that the dynamics can only be either a quotient of a complex torus or uniruled. In the general case, we will give a factorization result with respect to rational connectedness.

In $\S 3$, we will study the measure theoretic properties of dynamics. We will first construct invariant metrics and measures on bundles and subvarieties. We will conjecture several properties about these invariant measures: they can be obtained by iterations of smooth measures, or by probability measures of backward orbits of general points. We also conjecture that the Kobayashi pseudo-metrics vanish. We will prove some special of these properties using the works of Yau [59] and Briend-Duval [9].

In $\S 4$, we will study dynamics over number fields. We will first propose a dynamic Bogomolov conjecture and an equidistribution conjecture for dynamically generic small points. Following Chambert-Loir [16], we can make an equidistrubution conjecture on Berkovich's p-adic analytic spaces. Finally, we will prove that the equidistribution conjecture and Bogomolov conjecture are essentially equivalent to each other using a recent work of Yuan [60] on arithmetic bigness.

What should be, but is not, discussed in this article. Because of limitations of our time and knowledge, many interesting and important topics will not be treated in this article.

- First is the "real theory of dynamics". We prove some properties about the distribution of backward orbits but we say nothing about the forward orbits. Also we have zero knowledge about support of 
the canonical measure (which is actually crucial in our arithmetic theory). We have to learn from "real or $p$-adic experts of the dynamical system" about what we should do in the next step. We refer to Katok and Hasselblatt's book [32] for dynamics on manifolds, Milnor's book [41] for $\mathbb{P}^{1}$, Sibony's article [54] for $\mathbb{P}^{N}$, and Dinh-Sibony $[\mathbf{2 1}]$ for general complex variety respectively.

- The second topic is about the dynamics of correspondences and automorphisms of positive entropy. There are many beautiful examples that have been discovered and studied. For classification and complex theory of automorphisms of surfaces, in particular $K 3$ surfaces, we refer to Cantat $[\mathbf{1 5}, \mathbf{1 4}]$ and McMullen $[\mathbf{4 0}, \mathbf{3 9}]$. For arithmetic theory, we refer to the work of Autissier [2] for Hecke correspondences, Silverman [55] and Mazur [38] for involutions on K3 surfaces, and Kawaguchi [33] for some generalizations.

- The last topic is about the moduli of dynamical system. We will discuss the classification problem for which variety to have a dynamical system, and construction of dynamical system for moduli of subvarieties, but we will not study all polarized endomorphisms on a fixed variety. As the moduli of abelian varieties play a fundamental role in modern number theory and arithmetic geometry, it will be an interesting question to construct some interesting moduli spaces for dynamics. We refer to Silverman's paper [56] for the moduli of dynamics on $\mathbb{P}^{1}$.

Acknowledgments. This work grew out of a talk given at a memorial conference for S.S. Chern at Harvard University. I would like to thank S.T. Yau for this assignment so that I have the motivation to learn a lot of mathematics from the web in order to give a fair background on arithmetical dynamics. I would also like to thank Yuefei Wang and Yunping Jiang for explaining to me some common sense of the general dynamical system, and Antoine Chmbert-Loir, Xander Faber, Curtis McMullen, Bjorn Poonen, Nessim Sibony, Joe Silverman for their helpful comments on an early version of this paper. Finally, I would like to thank Xander Faber, Johan de Jong, Kathy O'Neil, and Xinyi Yuan for their patience in listening to my lectures during the initial preparation of the note. This work has been supported by the National Science Foundation of the USA and the Core Research Group of the Chinese Academy Sciences.

\section{Kähler and algebraic dynamics}

In this section, we will first give some basic definitions of polarized dynamics in Kähler geometry and algebraic geometry, and some basic categorical constructions, such as the fiber product and quotients. Then we will propose our first major conjecture: a dynamic Manin-Mumford conjecture. Finally we will list some examples, including abelian varieties, projective spaces, and the Chow variety of 0 -cycles. The main tools in this section 
are Serre's theorem on a Kähler analogue of the Weil conjecture, Deligne's theory on intersections of line bundles, and a conjectured Kähler analogue of the positivity of Deligne's pairing on the Chow variety.

\subsection{Endomorphisms with polarizations.}

Kählerian dynamical system. Let us first recall some definitions about Kähler manifolds. See [27] for details. Recall that a Kähler manifold is a complex manifold $X$ with a differential form $\omega$ of type $(1,1)$ such that $d \omega=0$ and that locally if we write

$$
\omega=i \sum h_{i, j} d z_{i} \wedge d \bar{z}_{j}
$$

then $\left(h_{i, j}\right)$ is a positive definite hermitian matrix. The form $\omega$ here is called a Kähler form and its class

$$
[\omega] \in H^{1,1}(X, \mathbb{R}):=H^{1,1}(X, \mathbb{C}) \cap H^{2}(X, \mathbb{R})
$$

is called a Kähler class.

By a Kähler variety $X$ with a Kähler form $\omega$ we mean an analytic variety which admits a finite map $f: X \rightarrow M$ to a Kähler manifold $M$ with a Kähler form $\eta$ such that $f^{*} \eta=\omega$.

Let $\phi: X \longrightarrow X$ be an endomorphism of a compact Kähler variety. Then $\phi$ acts on $H^{1,1}(X, \mathbb{R})$ by the pull-back $\phi^{*}$. We say that $\phi$ is polarizable by a Kähler class $\xi$ if

$$
\phi^{*} \xi=q \xi
$$

for some integer $q>1$. A polarized Kähler dynamical system is by definition a triple $(X, \phi, \xi)$ as above. The number $\operatorname{dim} X \cdot \log q$ is called the entropy of the dynamical system, and $\log q$ is called the entropy slope. One immediate fact about polarized endomorphisms is the following:

Lemma 1.1.1. Let $\phi: X \longrightarrow X$ be a polarized endomorphism. Then $\phi$ is finite with degree $\operatorname{deg} \phi=q^{\operatorname{dim} X}$.

Proof. Indeed, for any subvariety $Y$ in $X$, one has the formula

$$
\operatorname{deg}\left(\left.\phi\right|_{Y}\right) \int_{\phi(Y)} \omega^{\operatorname{dim} Y}=\int_{Y} \phi^{*} \omega^{\operatorname{dim} Y}=q^{\operatorname{dim} Y} \int_{Y} \omega^{\operatorname{dim} Y} \neq 0 .
$$

Here $\operatorname{deg}(\phi \mid Y)$ is defined to be 0 if $\operatorname{dim} \phi(Y)<\operatorname{dim} Y$. The above equation implies that $\operatorname{deg}\left(\left.\phi\right|_{Y}\right) \neq 0$. Taking $Y=\phi^{-1}(x)$, we get that $Y$ is finite. Thus $\phi$ is finite. Taking $Y=X$, we get that $\operatorname{deg}(\phi)=q^{\operatorname{dim} X}$.

A deep property of it is the following Kähler analogue of Weil's conjecture about eigenvalues of $\phi^{*}$ on cohomology:

THEOREM 1.1.2 (Serre [53]). Let $\phi: X \rightarrow X$ be a polarizable endomorphism of degree $q^{n}$. Then the eigenvalues of $\phi^{*}$ on each cohomology $H^{i}(X, \mathbb{R})$ have absolute value $q^{i / 2}$. 
Proof. Consider the cup product

$$
H^{i}(X, \mathbb{C}) \times H^{2 n-i}(X, \mathbb{C}) \longrightarrow H^{2 n}(X, \mathbb{C}) \simeq \mathbb{C} .
$$

Here the last map is given by integration. Let $\xi$ be a Kähler class such that $\phi^{*} \xi=q \xi$. Notice that $\xi^{n}$ is a generator of $H^{2 n}(X, \mathbb{C})$. So $\phi^{*}$ on $H^{2 n}(X, \mathbb{C})$ is given by multiplication by $q^{n}$. Now let $g$ denote the endomorphism on $H^{*}(X, \mathbb{C})=\oplus_{i} H^{i}(X, \mathbb{C})$ that has restriction $q^{-i / 2} \phi^{*}$ on $H^{i}(X, \mathbb{C})$. Then the above product is invariant under $g$, and so is the class $\xi$. Now we use the Hard Lefshetz theorem ([27], page 122) to give a decomposition of $H^{i}(X, \mathbb{C})$. For $i \leq n$, let $P_{i}(X)$ denote the kernel of the map

$$
H^{i}(X, \mathbb{C}) \longrightarrow H^{2 n-i+2}(X, \mathbb{C}), \quad \alpha \longrightarrow \xi^{n-i+1} \wedge \alpha .
$$

Then $H^{*}(X, \mathbb{C})$ is a direct sum of $\xi^{j} P_{i}$ with $i \leq n, i+2 j \leq 2 n$. Obviously, this decomposition is invariant under the action by $g$, and so it suffices to show that the eigenvalues of $g$ on $P_{i}$ have absolute value 1 . Moreover, by the Hodge index theorem (or Hodge and Riemann bilinear relations, [27], page 123) the pairing on $P_{i}$ defined by

$$
P_{i} \times P_{i} \longrightarrow \mathbb{C}, \quad(\alpha, \beta)=\int \alpha C(\beta) \kappa^{n-i}
$$

is positively definite. Here $C$ is an operator on $H^{*}(X, \mathbb{C})$ such that on the Hodge component $H^{p, q}$ with $p+q=i$, it is given by

$$
\alpha \mapsto(-1)^{(n-i)(n-i-1) / 2} \sqrt{-1}^{p-q} \bar{\alpha} .
$$

It is easily checked that $g$ is unitary with respect to this pairing. It follows that $g$ has eigenvalues with norm 1 . Thus the eigenvalues of $\phi^{*}$ have norm $q^{i / 2}$ on $H^{i}(X, \mathbb{C})$.

Endomorphisms with positive entropy. We say that a finite endomorphism $\phi$ of a compact Kähler variety has positive entropy if there is a semipositive class $\xi \in H^{1,1}(X, \mathbb{R})$ such that

$$
\phi^{*} \xi=q \xi
$$

with $q>1$. The notion of "positive entropy" here is equivalent to the same notion in the topological sense and to the statement that $\phi^{*}$ on $H^{1,1}(X, \mathbb{R})$ has an eigenvalue of absolute value greater than 1 . See Dinh-Sibony's paper [22] for details. The proof of Corollary 2.2 of that paper also shows that $\phi^{*}$ on $H^{p}(X, \mathbb{Z})$ has eigenvalues with absolute values bounded by the $p$-th power of the absolute value of the eigenvalues on $H^{1,1}(X, \mathbb{R})$. Notice that all eigenvalues on $H^{i}(X, \mathbb{Z})$ are algebraic integers with product a positive integer. Thus if $\phi$ does not have positive entropy then all of its eigenvalues on $H^{*}(X, \mathbb{Z})$ are roots of unity. Thus $\left(\phi^{*}\right)^{N}$ for some fixed $N$ has eigenvalues equal to 1 . This implies in particular that $\phi$ is the identity on $H^{2 n}(X, \mathbb{Z})$. Thus $\phi$ is a biholomorphic map. We conclude that if $\phi$ has zero entropy then $\phi$ is an automorphism. Notice that this statement is true for a dynamical system on a compact manifold. See Theorem 8.3.1 in Katok-Hasselblatt's book $[32]$. 
The category of dynamical system. We can define a morphism $f: \phi \rightarrow \psi$ of two endomorphisms $\phi: X \longrightarrow X$ and $\psi: Y \rightarrow Y$ as usual by a morphism $f: X \rightarrow Y$ such that $f \circ \phi=\psi \circ f:$

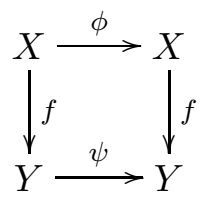

If $\phi$ and $\psi$ are polarized by classes $\xi$ and $\eta$ with the same entropy slope, then $\phi$ is polarized by all positive classes $\xi+c f^{*} \eta$ where $c \in \mathbb{R}$. Especially, if $f$ is finite and $\psi$ is polarized by $\eta$, then $\phi$ is polarized by $f^{*} \eta$.

If $f$ is proper and flat with relative dimension $d$, and $\phi$ is polarized by a class $\xi$ with entropy slope $\log q$, then we claim that $\psi$ is polarized by the form

$$
\eta:=\int_{X / Y} \xi^{d+1}
$$

with the same entropy slope $\log q$ as $\phi$ provided that $\eta$ is a Kähler class on $Y$. See Conjecture 1.2.3 and Remark 1.2.4. below. Indeed, for any point $y \in Y$, in the diagram

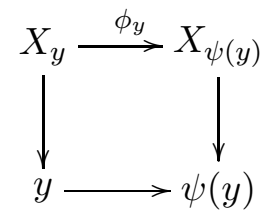

the morphism $\phi_{y}$ has degree $q^{d}$ as $\int_{X_{y}} \xi^{d}>0$, and

$$
\operatorname{deg}\left(\phi_{y}\right) \int_{X_{\psi(y)}} \xi^{d}=\int_{X_{y}} \phi^{*} \xi^{d}=q^{d} \int_{X_{y}} \xi^{d}
$$

It follows that

$$
\left(\psi^{*} \eta\right)(y)=\int_{X_{\psi(y)}} \xi^{d+1}=\frac{1}{\operatorname{deg} \phi_{y}} \int_{X_{y}} \phi^{*} \xi^{d+1}=q \int_{X_{y}} \xi^{d+1}=q \eta
$$

Especially, if $f$ is finite and flat, then $\phi$ is polarized if and only if $\psi$ is polarized. One application is the normalization $f: \widetilde{X} \longrightarrow X$ : obviously $\phi$ induces an endomorphism $\widetilde{\phi}$ of $\widetilde{X}$ which is polarized by the class $f^{*} \xi$.

We say two endomorphisms $\phi, \psi: X \longrightarrow X$ are equivalent if there are positive numbers $m, n$ such that $\phi^{m}=\psi^{n}$. We will mainly study the properties of endomorphisms depending only on their equivalence classes. Thus it makes sense to define the entropy class for the equivalence class of an endomorphism $\phi$ to be

as a $\mathbb{Q}$-line in $\mathbb{R}$.

$$
\mathbb{Q} \log (\operatorname{deg} \phi)
$$

Notice that the product of two polarized endomorphisms may not be polarizable. If we allow to replace them by equivalent ones, then a sufficient 
condition is that they have the same entropy class. More precisely, let $\phi: X \longrightarrow X$ and $\psi: Y \longrightarrow Y$ be two endomorphisms of compact Kähler varieties polarized by $\omega \in H^{1,1}(X)$ and $\eta \in H^{1,1}(Y)$. The following two statements are equivalent:

(1) the endomorphism

$$
\phi \times \psi: X \times Y \longrightarrow X \times Y
$$

is polarizable by $\pi_{1}^{*} \omega+\pi_{2}^{*} \eta$ where $\pi_{i}$ are projections from $X \times Y$ to $X$ and $Y$;

(2) $\phi$ and $\psi$ have the same entropy slope.

If $f_{1}: \phi_{1} \rightarrow \psi$ and $\phi_{2} \rightarrow \psi$ are two morphism from two dynamic systems $X_{1}, X_{2}$ to a variety $Y$, then we can form the fiber product

$$
\phi_{1} \times_{Y} \phi_{2}: X_{1} \times_{Y} X_{2} \longrightarrow X_{1} \times_{Y} X_{2} .
$$

If they have the same entropy slope, then the product is again a polarized dynamical system in an obvious way.

Algebraic dynamical system. We now consider an endomorphism $\phi$ : $X \longrightarrow X$ of projective varieties. We may define algebraic polarization by replacing (1,1)-classes by line bundles. Let Pic $(X)$ denote the group of line bundles on $X$ which is isomorphic to $H^{1}\left(X, \mathcal{O}_{X}\right)$ and let $\operatorname{Pic}^{0}(X)$ denote the subgroup of line bundles which are algebraically equivalent to 0 , and let $N S(X)$ denote the quotient $\operatorname{Pic}(X) / \operatorname{Pic}^{0}(X)$ which is called the NeronSeveri group. Then the exact sequence

$$
0 \longrightarrow \mathbb{Z} \longrightarrow \mathcal{O}_{X} \longrightarrow \mathcal{O}_{X}^{\times} \longrightarrow 0
$$

induces the following natural isomorphisms:

$$
\mathrm{NS}(X) \simeq H^{1,1}(X, \mathbb{C}) \cap H^{2}(X, \mathbb{Z}), \quad \operatorname{Pic}^{0}(X) \simeq H^{1}\left(X, \mathcal{O}_{X}\right) / H^{1}(X, \mathbb{Z}) .
$$

Recall that a line bundle $\mathcal{L}$ is ample if some positive power $\mathcal{L}^{m}$ is isomorphic to the pull-back of the hyperplane section bundle for some embedding $i: X \longrightarrow \mathbb{P}^{N}$. By Kodaira's embedding theorem, $\mathcal{L}$ is ample if and only if its class in $N S(X) \subset H^{1,1}(X, \mathbb{R})$ is a Kähler class.

Let $\phi: X \longrightarrow X$ be an endomorphism of a projective variety. Then $\phi$ acts on Pic $(X)$. We say that $\phi$ is polarizable by a line bundle $\mathcal{L}$ (resp. $\mathbb{R}$-line bundle $\mathcal{L} \in \operatorname{Pic}(X) \otimes \mathbb{R})$ if

$$
\phi^{*} \mathcal{L} \simeq \mathcal{L}^{q}
$$

for some $q>0$.

An endomorphism $\phi: X \longrightarrow X$ of projective variety polarized by a line bundle $\mathcal{L}$ will be polarized by an integral Kähler class: we just take

$$
\xi=c_{1}(\mathcal{L}) \in H^{1,1}(X, \mathbb{Z}) .
$$

We want to show the converse is true: 
Proposition 1.1.3. Let $\phi: X \longrightarrow X$ be an endomorphism of smooth projective variety with a polarization by a Kähler class $\xi$ such that $\xi$ is integral, and that $\phi^{*} \xi=q \xi$ with $q$ integral and $>1$. Then there are line bundles $\mathcal{L}$ with class $\xi$ such that

$$
\phi^{*} \mathcal{L} \simeq \mathcal{L}^{q} .
$$

Proof. Let $\operatorname{Pic}^{\xi}(X)$ denote the variety of line bundles on $X$ with class $\xi$. Then we have a morphism

$$
\lambda: \quad \operatorname{Pic}^{\xi}(X) \longrightarrow \operatorname{Pic}^{0}(X), \quad \lambda(\mathcal{L})=\phi^{*} \mathcal{L} \otimes \mathcal{L}^{-q} .
$$

Notice that $\operatorname{Pic}^{\xi}(X)$ is a principal homogenous space of $\operatorname{Pic}^{0}(X)$. The induced homomorphism on $H^{1}$ 's is an endomorphism on $H^{1}(X, \mathbb{Z})$ given by $\lambda:=\phi^{*}-q$. By Proposition 1.1.2, all eigenvalues of $\phi^{*}$ on $H_{1}(X, \mathbb{Z})$ have eigenvalues with absolute values $q^{1 / 2}$. It follows from the assumption that $\lambda$ is finite and thus surjective. In particular we have an $\mathcal{L} \in \operatorname{Pic}^{\xi}(X)$ such that $\lambda(\mathcal{L})=0$. In other words $\phi^{*} \mathcal{L}=\mathcal{L}^{q}$.

Category of algebraic dynamical system. In the same manner as in Kähler case, we may define the morphism $f: \phi \rightarrow \psi$ between two endomorphisms of projective varieties $X, Y$. If $\phi$ and $\psi$ are both polarized by line bundles $\mathcal{L}$ and $\mathcal{M}$ with the same entropy slope, then $\phi$ is also polarized by any positive class of the form $\mathcal{L} \otimes f^{*} \mathcal{M}^{n}$. If $f$ is flat of relative dimension $d$, and $\phi$ is polarized by a line bundle $\mathcal{L}$, then $\psi$ is polarized by the following Deligne's pairing ([19], See also $[\mathbf{6 3}])$ :

$$
\int_{X / Y} \mathcal{L}^{\langle d+1\rangle}:=\langle\mathcal{L}, \ldots, \mathcal{L}\rangle .
$$

For convenience to reader, let us recall the definition. Let $\pi: \quad Z \longrightarrow C$ be a flat family of projective varieties of pure relative dimension $d$. Let $\mathcal{L}_{0}, \ldots \mathcal{L}_{d}$ be line bundles on $Z$. The Deligne pairing $\left\langle\mathcal{L}_{0}, \ldots, \mathcal{L}_{d}\right\rangle$ is a line bundle on $C$ which is locally generated by a symbol $\left\langle\ell_{0}, \ldots \ell_{d}\right\rangle$ modulo a relation, where $\ell_{i}$ are sections of $\mathcal{L}_{i}$ such that their divisors $\operatorname{div}\left(\ell_{i}\right)$ have empty intersection on fibers of $f$. The relation is given as follows. If $\alpha$ is a function, and $i$ is an index between 0 and $d$ such that $\operatorname{div}(\alpha)$ has disjoint intersection $Y:=\prod_{j \neq i} \operatorname{div}\left(\ell_{j}\right)$, then $Y$ is finite over $C$, and

$$
\left\langle\ell_{0}, \ldots, \alpha \ell_{i}, \ldots, \ell_{d}\right\rangle=\mathrm{N}_{Y}(\alpha)\left\langle\ell_{0}, \ldots, \ell_{d}\right\rangle .
$$

Here $\mathrm{N}_{Y}(\alpha)$ is the usual norm map $\mathrm{N}_{Y}: \pi_{*} \mathcal{O}_{Y} \longrightarrow \mathcal{O}_{C}$.

We may also define the polarized product or fiber product for polarized endomorphisms with the same entropy slope in the same manner as in Kähler case.

1.2. Preperiodic subvarieties. Let $\phi: X \longrightarrow X$ be an endomorphism of Kähler variety with a polarization. Let $Y$ be an analytic subvariety of $X$. We say that $Y$ is periodic if for some $k>0, \phi^{k}(Y)=Y$, and preperiodic if for some $m, \phi^{m}(Y)$ is periodic. Equivalently, $Y$ is preperiodic if the orbits of $Y$ under $\phi$ are finite. When $X$ is projective, it shown by Fakhruddin 
([23], Corollary 2.2) that for some $\ell, m \geq 1$ such that the system $\left(X, \phi, \mathcal{L}^{m}\right)$ can be extended to a dynamic system of $\mathbb{P}^{N}$, where $N=\operatorname{dim} \Gamma\left(X, \mathcal{L}^{m}\right)$.

If $Y$ is periodic, say $\phi^{k}(Y)=Y$. Then the restriction of $\phi^{k}$ on $Y$ is still polarized with entropy slope $k \log q$. The aim of our paper is to study the distribution properties of the set Prep $(X)$ of preperiodic points of $X$ in various topology. In this section we mainly focus on Zariski topology. Our first basic conjecture is the following:

Conjecture 1.2.1 (Dynamic Manin-Mumford). A subvariety $Y$ of $X$ is preperiodic if and only if $Y \cap \operatorname{Prep}(X)$ is Zariski dense in $Y$.

Dynamic topology. For a better understanding of the nature of the dynamic Manin-Mumford conjecture, it is helpful to introduce the following socalled dynamic topology on a dynamical system $(\phi, X, \xi)$ in which all closed sets are preperiodic subvarieties. To see it is really a topology, we check that the intersections of preperiodic subvarieties are still preperiodic. In this topology, the set of minimal subvarieties are exactly the set of preperiodic points. The conjecture 1.2.1 is equivalent to the following two statements:

(1) The preperiodic points on any preperiodic subvariety are Zariski dense; when $X$ is projective, this is actually a Fakhruddin [23], Theorem 5.1.

(2) On Prep $(X)$, dynamic topology = Zariski topology.

The Zariski closure of preperiodic points in a preperiodic subvariety is again preperiodic. Thus for the first statement it suffices to consider periodic points in the periodic subvariety.

Conjecture 1.2.2. Let $Y$ be a periodic subvariety of dimension $r$ : $\phi^{m} Y=Y$ for some $m>0$. Then as $k \longrightarrow \infty$,

$$
\#\left\{y \in Y, \phi^{k m}(x)=x\right\}=q^{r k m}(1+o(1)) .
$$

By Serre's Theorem 1.1.2, the conjecture is true if $Y$ is smooth, polarizable, and if most of the fixed points have multiplicity one. Indeed, in this case without loss of generality we may simply assume that $Y=X$ and that $a=1$. For any fixed point $x$ of $\phi^{k}$ the multiplicity $m_{k}(x)$ is defined to be the length of the dimension of the maximal quotient of the local $\mathcal{O}_{X, x}$ where the action of $\left(\phi^{k}\right)^{*}$ is trivial:

$$
m_{k}(x):=\operatorname{dim}_{\mathbb{C}} \mathcal{O}_{X, x} /\left(\left(\phi^{k}\right)^{*}-1\right) \mathcal{O}_{X, x} .
$$

We define $m_{k}(x)=0$ if $x$ is not a fixed point of $\phi^{k}$. Then by Lefshetz fixed point theorem $([\mathbf{2 7}]$, page 421$)$, the left hand side is

$$
\sum_{x} m_{k}(x)=\sum_{i}(-1)^{i} \operatorname{tr}\left(\left(\phi^{k}\right)^{*}: H^{i}(X, \mathbb{C})\right) \text {. }
$$

By Theorem 1.1.2, the right hand has estimate $q^{k n}$ if $\phi$ is polarizable.

A consequence of Conjecture 1.2.2. is that the set of pre-periodic points of $X$ is countable. This is the true for general preperiodic subvarieties proved Corollary 1.2.7. 
Dynamical systems of subvarieties. In the following, we will introduce some dynamical system on the Chow variety. Notice that the Chow variety is not of finite type; it is a union of a countably many subvarieties of finite type. Later on, we will construct some dynamic systems on subvarieties of Chow variety of finite type which are conjectured to be the Zariski closure of periodic subvarieties.

Let us start with a compact Kähler variety $X$ with a Kähler class $\xi$. Let $\mathcal{C}(X)$ denote the variety of cycles on $X$ with pure dimension [4]. Then $\mathcal{C}(X)$ is a union of countably many Kähler varieties. We call $\mathcal{C}(X)$ the Chow variety of $X$, as when $X$ is projective, $\mathcal{C}(X)$ is simply the usual Chow variety of $X$.

We may equip $\mathcal{C}(X)$ with the structure of a Kähler variety as follows. Let

$$
(i, \pi): \mathcal{Z}(X) \longrightarrow X \times \mathcal{C}(X)
$$

be the universal family of cycles. For each $d$ between 0 and $n$ let $\pi_{d}$ : $\mathcal{Z}_{d}(X) \rightarrow \mathcal{C}_{d}(X)$ denote the moduli of cycles of pure dimension $d$. Then for any Kähler class $\xi$ of $X$, we define

$$
\eta_{d}:=\int_{\mathcal{Z}_{d}(X) / \mathcal{C}_{d}(X)}\left(i^{*} \xi\right)^{d+1} \in H^{1,1}\left(\mathcal{C}_{d}(X)\right) .
$$

Conjecture 1.2.3. The class $\eta_{d}$ is a Kähler class on $\mathcal{C}_{d}(X)$.

REMARKS 1.2.4.

(1) This conjecture implies that for any flat morphism of compact Kähler manifold $f: X \rightarrow Y$ and any Kähler class $\xi$ on $X$, the class $\eta=\int_{X / Y} \xi^{d+1}$ is a Kähler on $Y$. Indeed, in this case we have an embedding $Y \longrightarrow \mathcal{C}_{d}(X)$ for $d$ the relative dimension of $f$.

(2) The conjecture is true when both $X$ and $Y$ are projective varieties and when $\xi$, the first Chern class of an ample line bundle on $X$. Indeed, in this case replacing $\mathcal{L}$ by a power we may assume that $\mathcal{L}=i^{*} \mathcal{O}(1)$ for some embedding $X \longrightarrow \mathbb{P}^{n}(\mathbb{C})$; then $\eta=j^{*} \mathcal{O}(1)$ for some embedding $j: Y \longrightarrow \mathbb{P}^{N}(\mathbb{C})$. See $[63]$.

If $\phi: X \longrightarrow X$ is an endomorphism polarized by a positive class $\xi$ then $\phi$ induces an endomorphism

$$
\phi_{d}: \mathcal{C}_{d}(X) \longrightarrow \mathcal{C}_{d}(X), \quad \phi_{d}(Z)=\phi_{*}(Z)=\operatorname{deg}\left(\left.\phi\right|_{Z}\right) \cdot \phi(Z) .
$$

It clear that $\phi_{d}$ is polarized by $\eta_{d}$ :

$$
\phi^{*}\left(\eta_{d}\right)=q^{d+1} \cdot \eta_{d}
$$

In the following, we want to construct countably many subvarieties $\mathcal{C}(d, \gamma, k)$ of $\mathcal{C}_{d}(X)$ of finite type and endomorphisms $\phi_{d, \gamma, k}$ with polarizations such that every periodic subvariety is represented by points in $\mathcal{C}(d, \gamma, k)$.

First we may decompose $\mathcal{C}_{d}(X)$ further as a union of closed subvarieties $\mathcal{C}(d, \delta)$ representing cycles with degree $\delta$. 
Recall that for an integral subvariety $Z$,

$$
\phi_{*}(Z)=\left.\operatorname{deg} \phi\right|_{Z} \cdot \phi(Z) .
$$

The class $\gamma^{\prime}:=[\phi(Z)]$. Compute the degree to obtain

$$
\operatorname{deg}(\phi(Z))=q^{d} \frac{\operatorname{deg}(Z)}{\left.\operatorname{deg} \phi\right|_{Z}} .
$$

If furthermore $Z$ is fixed by some positive power $\phi^{k}$ of $\phi$, then the above implies that

$$
\left.\operatorname{deg} \phi^{k}\right|_{Z}=q^{k d} .
$$

For each positive integer $k$, let $\mathcal{C}(d, \delta, k)$ denote the subvariety of $\mathcal{C}(d, \delta)$ of cycles $Z$ of degree $\delta$ such that

$$
q^{-k d \ell}\left(\phi_{*}^{k \ell} Z\right), \quad \ell=1,2, \ldots
$$

are all integral. Then we can define an endomorphism

$$
\phi_{d, \delta, k}: \mathcal{C}(d, \delta, k) \longrightarrow \mathcal{C}(d, \delta, k), \quad Z \longrightarrow q^{-k d}\left(\phi_{*}^{k} Z\right) .
$$

Proposition 1.2.5. The endomorphisms $\phi_{d, \gamma, k}$ are all polarized with respect to the bundle $\eta_{d}$ with entropy slope $k \log q$ :

$$
\phi_{d, \delta, k}^{*} \eta_{d}=q^{k} \cdot \eta_{d}
$$

Proof. By integration over fibers over $Z$ for the form $\xi^{d+1}$, we have

$$
\phi_{d, \delta, k}^{*} \eta_{d}=\frac{q^{(d+1) k}}{q^{d k}} \cdot \eta_{d}=q^{k} \eta_{d}
$$

In view of Conjecture 1.2.1 for $\mathcal{C}(d, \delta, k)$ we have the following:

Conjecture 1.2.6. The variety $\mathcal{C}(d, \delta, k)$ is the Zariski closure of points in $\mathcal{C}(X)$ representing periodic cycles $Y$ of $X$ such that the following identities hold:

$$
\operatorname{dim} Y=d, \quad \operatorname{deg} Y=\delta, \quad \phi^{k}(Y)=Y .
$$

When $X$ is projective, this conjecture is a theorem of Fakhruddin [23].

Notice that each periodic subvariety represents a fixed point in some $\phi_{d, \gamma, k}^{*}$. Thus they are finite in each $\mathcal{C}(d, \gamma, k)$. In other words, the set of preperiodic subvarieties of $X$ is countable.

Corollary 1.2.7. Let $\phi: X \longrightarrow X$ be an endomorphism of a compact Kähler manifold with a polarization. Then the set of preperiodic subvarieties of $X$ is countable.

From the known example, it seems that all irreducible preperiodic subvarieties of $X$ have the bounded geometry, i.e., lie in a finite union of components of the Chow variety $\mathcal{C}(X)$. The following is a reformulation of the question: 
Question 1.2.8. Does there exist a number $\delta$ such that

$$
\int_{Y} \xi^{d} \leq \delta
$$

for any irreducible preperiodic subvariety $Y$ of dimension $d$ ?

If $X$ is polarized by line bundles, then we may replace the above integrals by Deligne's pairing in $\S 1.1$. Thus we will naturally define line bundles on $\mathcal{C}_{d}(X)$ denoted by

$$
\mathcal{L}^{\langle d+1\rangle}:=\left\langle i^{*} \mathcal{L}, \ldots, i^{*} \mathcal{L}\right\rangle=\text { Deligne pairing of } d+1 \mathcal{L} \text { 's }
$$

whose Chern class is equal to

$$
\int_{\mathcal{Z}_{d}(X) / \mathcal{C}_{d}(X)} c_{1}(\mathcal{L})^{d+1} .
$$

1.3. Examples. In this subsection we want to give some examples of endomorphisms with polarizations.

Complex torus. Our first example is the complex torus $X=\mathbb{C}^{n} / \Lambda$ where $\Lambda$ is a lattice in $\mathbb{C}^{n}$ with Kähler class

$$
\xi=i \sum d z_{i} \wedge d \bar{z}_{i}
$$

and $\phi$ is given by multiplication by an integer $m>1$. Then we have

$$
\phi^{*} \xi=q \xi, \quad q=m^{2} .
$$

In this case the preperiodic points are exactly the torsion points:

$$
\operatorname{Prep}(X)=\Lambda \otimes \mathbb{Q} / \Lambda \text {. }
$$

The conjecture 1.2.2 is trivial: the set of fixed points by $\phi^{k}$ is the set of torsion points $X\left[m^{k}-1\right]$ which has cardinality

$$
\left(m^{k}-1\right) 2 n=q^{n k}+O\left(q^{(n-1) k}\right) .
$$

The preperiodic subvarieties are translations of abelian subvarieties by torsion points. When $X$ is an abelian variety, the dynamic Manin-Mumford conjecture is the original Mumford-Manin conjecture proved firstly by Raynaud [52]: Let $Y$ be a subvariety of $X$ which is not a translate of an abelian subvariety. Then all the torsion points on $Y$ are included in a proper subvariety. There are other proofs $([\mathbf{6 4}, \mathbf{1 8}])$, but all of them uses heavily the algebraic property (or even arithmetic property) of $X$. Thus they can't be generalized directly to the general complex torus.

Projective spaces. Let $X=\mathbb{P}^{n}$, and $\phi: X \longrightarrow X$ be any map of degree $d>1$ defined by $n+1$-homogenous polynomials of degree $q$ with no non-trivial common zeros. By Fakhruddin's result ([23], Corollary 2.2), any polarized dynamic system is a subsystem of a certain system on $\mathbb{P}^{n}$. Conjecture 1.2.2 looks easy but I don't know how to prove it. In the simplest case $\phi\left(x_{0}, \ldots x_{n}\right)=\left(x_{0}^{m}, \ldots, x_{n}^{m}\right)$ where $m \neq \pm 1$, the preperiodic points are exactly the points where the coordinates $x_{i}$ are either 0 or roots of unity. This is a multiplicative group analogous situation of abelian varieties: $\mathbb{P}^{n}$ 
is the union of multiplicative groups defined by the vanishing of some coordinates. The Manin-Mumford conjecture is true! See Lang [36], page 207, Ihara-Serre-Tate for $n=2$, and Laurent [37] for the general case. On each multiplicative group, the conclusions are the same as in the abelian varieties case.

The next nontrivial work is when $X=\mathbb{P}^{1} \times \mathbb{P}^{1}, \phi=\left(\phi_{1}, \phi_{2}\right)$. The conjecture is true when Julia sets of $\phi_{i}$ are very different $([\mathbf{4 2}])$. Any curve $C$ in $\mathbb{P}^{1} \times \mathbb{P}^{1}$ which is neither horizontal nor vertical contains at most finitely many preperiodic points.

Weighted projective spaces. Fix an $n+1$-tuple of positive integers $r=$ $\left(r_{0}, \ldots, r_{n}\right)$. Then we have an action of $\mathbb{C}^{\times}$on $\mathbb{C}^{n+1} \backslash\{0\}$ by

$$
\left(z_{0}, \ldots, z_{n}\right) \mapsto\left(t^{r_{0}} z_{0}, \ldots, t^{r_{n}} z_{n}\right), \quad\left(t \in \mathbb{C}^{\times}\right) .
$$

The quotient is called a called a weighted projective space and denoted by $\mathbb{P}_{r}^{n}$. Notice that $\mathbb{P}_{r}^{n}$ is a projective space and can be defined by Proj $\mathbb{C}\left[z_{0}, \ldots\right.$, $\left.z_{n}\right]_{r}$ where $\mathbb{Z}\left[z_{0}, \ldots, z_{n}\right]_{r}$ is the graded algebra $\mathbb{Z}\left[z_{0}, \ldots, z_{n}\right]$ with weighted degree $\operatorname{deg} z_{i}=r_{i}$. Any endomorphism of $\mathbb{P}_{r}^{n}$ is again given by homogenous polynomials with nontrivial zeros and with the same degree, say $q$, and is polarized by the the bundle $\mathcal{O}(1)$. Notice that $\mathbb{P}_{r}^{n}$ is in general a singular variety and is a quotient of $\mathbb{P}^{n}$ by the diagonal action the product of roots of $r_{i}$-th roots of unity:

$$
\mu_{r_{0}} \times \cdots \mu_{r_{n}}
$$

Thanks to N. Sibony who showed this example to me!

Dynamical projective bundles. Let $\phi: \quad X \longrightarrow X$ be an endomorphism of compact Kähler variety polarized by a Kähler class $\xi$ of entropy slope $\log q$. Let $\mathcal{L}_{i}(i=1, \ldots, n)$ be line bundles on $X$ such that

$$
\psi_{i}: \quad \phi^{*} \mathcal{L}_{i} \simeq \mathcal{L}_{i}^{q}
$$

Define a vector bundle $\mathcal{V}$ as follows:

$$
\mathcal{V}=\mathcal{L}_{0} \oplus \mathcal{L}_{1} \oplus \cdots \oplus \mathcal{L}_{n_{1}}
$$

and define $Y$ to be the corresponding projective bundle:

$$
Y=\mathbb{P}(\mathcal{V}) \text {. }
$$

Then $\psi_{i}$ induces embeddings of vector bundles

$$
\phi^{*} \mathcal{V} \longrightarrow \operatorname{Sym}^{q} \mathcal{V}
$$

Thus we have an endomorphism

$$
f: \mathbb{P}(\mathcal{V}) \longrightarrow \mathbb{P}(\mathcal{V})
$$

such that

$$
f^{*} \mathcal{O}_{\mathbb{P}(\mathcal{V})}(1)=\mathcal{O}_{\mathbb{P}_{X}(\mathcal{V})}(q)
$$

Then $f$ is polarizable by bundles $c_{1}\left(\mathcal{O}_{\mathbb{P}(\mathcal{V})}(1)\right)+m \xi$ which is positive on $\mathbb{P}(\mathcal{V})$ when $m \gg 0$. We don't know if Conjecture 1.2.1 is true or not on $Y=\mathbb{P}_{X}(\mathcal{V})$ if it is already true on $X$. A typical example is when $X=A$ is 
an abelian variety, $\phi=[n]$ for some $n>1, q=n^{2}$, and $\mathcal{L}_{i}(i=1, \ldots, n)$ are line bundles such that $\mathcal{L}_{i}$ are ample and symmetric:

$$
[-1]^{*} \mathcal{L}_{i} \simeq \mathcal{L}_{i}
$$

Another case is when $\mathcal{L}_{i}$ are torsion bundles. Then we will have $[n]^{*} \mathcal{L}_{i} \simeq$ $\mathcal{L}_{i}^{n^{2}}$ if $\mathcal{L}_{i}^{n-1}=\mathcal{O}_{X}$ for all $i$. This case corresponds to the almost split semiabelian variety. The conjecture 1.2 .1 is true by Chambert-Loir $[\mathbf{1 7}]$.

Chow variety of 0-cycles. Let $\phi: X \longrightarrow X$ be an endomorphism with a polarization. Let $\delta$ be a positive number. Then the Chow variety $\mathcal{C}(0, \delta)$ of zero cycles of degree $\delta$ has an endomorphism $\phi_{0}$ which is polarized by classes $\eta_{0}$. Recall that $\eta_{0}$ is defined as $\pi_{*} i^{*} \xi$, where $(i, \pi)$ is the embedding of universal $\delta$-cycles $\mathcal{Z}(0, \delta) \longrightarrow \mathcal{C}(0, \delta) \times X$.

Here is situation of curves: If $X=\mathbb{P}^{1}$, then $\mathcal{C}_{\delta}=\mathbb{P}^{\delta}$. If $C$ is an elliptic curve, then $\mathcal{C}_{d}$ is a $\mathbb{P}^{\delta-1}$ bundle over $E$.

\section{Classifications}

In the following we want to discuss some classification problems for the dynamical system. We will first study the first Chern class for smooth $d y$ namics and classify them when the first Chern class vanishes using a result of Beauville. Then we show that the smooth dynamics is uniruled in the remaining case using results of Miyaoka-Mori on a criterion on uniruledness and Bouchson-Demailly-Paum-Peternell on a criterion on pseudoeffectiveness. Using a result of Miyaoka-Mori and Campana, we will also give a fiberation decomposition with respect to the rational connectedness for general dynamics. Finally we give a full classification for which surface admits a polarized endomorphism using work of Fujimoto and Nakayama.

2.1. Positivity of the first Chern class. First notice that for any dynamical system $X$, the canonical class can't be positive when $X$ is smooth.

Proposition 2.1.1 (Fakhruddin [23], Theorem 4.2 for $X$ projective). Let $\phi: X \longrightarrow X$ be an endomorphism of a compact Kähler manifold with a polarization by a class $\xi$. Let $K_{X}$ be the canonical class of $X$. Let $R_{\phi}$ be the ramification divisor of $\phi$. Then the following statements hold:

$$
(1-q) \xi^{n-1} \cdot K_{X}=\xi^{n-1} \cdot R_{\phi}
$$

(2) The Kodaira dimension of $X$ is $\leq 0$.

(3) If $c(X)=-c_{1}\left(K_{X}\right)=0$ in $H^{1,1}(X, \mathbb{Z})$, then $X$ has an etale cover by complex torus:

$$
X \simeq T / G, \quad T=\mathbb{C}^{n} / \Lambda,
$$

where $\Lambda$ is a full rank Lattice in $\mathbb{C}^{n}$, and $G$ is a finite group acting on $T$ without fixed points. Moreover the endomorphism $\phi$ is induced by a linear endomorphism $\widetilde{\phi}$ on $\mathbb{C}^{n}$ as a $\mathbb{C}$-vector space. 
Proof. By definition of ramification divisor,

$$
K_{X}=\phi^{*} K_{X}+R_{\phi} .
$$

Thus

$$
\begin{aligned}
\xi^{n-1} \cdot R_{\phi} & =\xi^{n-1} \cdot K_{X}-\xi^{n-1} \cdot \phi^{*} K_{X} \\
& =\xi^{n-1} \cdot K_{X}-q^{1-n} \phi^{*}\left(\xi^{n-1} \cdot K_{X}\right) \\
& =\xi^{n-1} \cdot K_{X}-q \cdot \xi^{n-1} \cdot K_{X}=(1-q) \xi^{n-1} \cdot K_{X} .
\end{aligned}
$$

This proves the first part of the proposition.

If the Kodaira dimension of $X$ is positive, then some multiple of $K_{X}$ is effective and nonzero; then $\xi^{n-1} K_{X}>0$. As $R_{\phi}$ is effective and $q>0$, we thus have a contradiction! So we have proved the second part.

If $c_{1}(X)=-c_{1}\left(K_{X}\right)=0$, then both sides are zero in the equation in Part 1. Thus $R_{\phi}=0$ and $\phi$ is unramified. In this case $\phi$ is induced from an unramified automorphism $\widetilde{\phi}$ of the universal covering $\widetilde{\widetilde{X}}$. Now we apply a theorem of Beauville ([5], Theorem 1, page 759) that $\widetilde{X}$ is isomorphic to $\mathbb{C}^{k} \times M$ where $M$ is a simply connected Kähler manifold and the pull-back $\widetilde{\xi}$ on $\widetilde{X}$ of $\xi$ is a sum $\widetilde{\xi}=\kappa+\eta$, where $\kappa$ is a flat Kähler class on $\mathbb{C}^{k}$,

$$
\kappa=\sqrt{-1} \sum_{j=1}^{k} a_{j} d z_{j} d \bar{z}_{j},
$$

and $\eta$ is a class on $M$. Since all morphisms from $M$ to $\mathbb{C}^{k}$ are constant, the morphism $\widetilde{\phi}$ has the form

$$
\widetilde{\phi}(z, m)=\left(u(z), v_{z}(m)\right) .
$$

Here $u(z)$ is an automorphism of $\mathbb{C}^{k}$ and $v_{z}$ is an automorphism of $M$ for each given $z \in \mathbb{C}^{k}$. Now we apply the equality $\widetilde{\phi}^{*} \widetilde{\xi}=q \widetilde{\xi}$ to conclude that $v_{z}^{*} \eta=q \eta$. As $M$ is compact and $q>1$ and $\eta$ is positive, this is impossible unless $M$ is a point. Thus we have shown that $\widetilde{X}=\mathbb{C}^{k}$. By the same theorem in [5], $X$ is then an unramified quotient of a complex torus $T=\mathbb{C}^{n} / \Lambda$ and $\Lambda$ is invariant under the action by $\widetilde{\phi}$.

To show the last statement, we need only to show that the induced endomorphism $\psi$ on $T=\mathbb{C}^{n} / \Lambda$ has a fixed point. We will use the Lefshetz fixed point theorem. Notice that $\psi$ is again polarized. Thus the eigenvalues $\lambda_{i}$ of $\psi^{*}$ on $H^{1}(T, \mathbb{Z})$ are greater than 1 by Theorem 1.1.2. Notice that $H^{i}(X, \mathbb{Z})$ is the $i$-th exterior power of $H^{1}(X, \mathbb{Z})$, and thus the eigenvalues of $\psi^{*}$ are given by $\lambda_{j_{1}} \cdots \lambda_{j_{i}}$ of $i$ distinct elements in $\lambda_{i}$ 's. Thus the number of fixed points with multiplicity is given by

$$
\sum_{i}(-1)^{i} \operatorname{tr}\left(\psi^{*}, H^{i}(X, \mathbb{Z})\right)=\sum_{j_{1}, \ldots, j_{k}}\left(-\lambda_{j_{1}}\right) \cdots\left(-\lambda_{j_{k}}\right)=\prod\left(1-\lambda_{i}\right) .
$$

As $\left|\lambda_{i}\right|>1$, the above number is nonzero. Thus $\psi$ has a fixed point. After changing coordinates, we may assume that $\psi$ and $\widetilde{\phi}$ fix the origin. 
2.2. Uniruledness. By Proposition 2.1.2, the classification of smooth dynamical systems is reduced to the case where $X$ has Kodaira dimension $-\infty$. If $n=\operatorname{dim} X=1$, then $X$ is simply $\mathbb{P}^{1}$. Later on we will discuss the case of surfaces. In general, it is conjectured that a Kähler manifold with Kodaira dimension $-\infty$ is always uniruled, i.e., covered by rational curves. The conjecture is true for projective varieties of $\operatorname{dim} \leq 3$ by Mori [44], and non-algebraic Kähler manifolds of dimensions $\leq 3$ with possibly the exception of simple threefolds by Peternell [48]. In the following we want to prove the uniruledness in the smooth and projective case using a ruledness criterion of Miyaoka-Mori [43] and a pseudo-effectiveness criterion of Bouchson, Demailly, Paum, and Peternell [51].

Proposition 2.2.1. Let $\phi: X \longrightarrow X$ be an endomorphism of a projective manifold with a polarization by line bundles $\mathcal{L}$. Assume that $c_{1}(X) \neq 0$. Then $X$ is uniruled.

Proof. Let $\xi$ be the corresponding Kähler class of $\mathcal{L}$. By the first part of Proposition 2.1.1, $\xi^{n-1} K_{X} \leq 0$. If $K_{X} \cdot \xi^{n-1}<0$, then since $\mathcal{L}$ is ample, $K_{X}$ has negative intersection with strongly movable curves as in [51]. By Theorem 0.2 in [51], $K_{X}$ is not pseudo-effective, i.e., $c_{1}\left(K_{X}\right)$ is not in the closure of the cone in $H^{1,1}(X, \mathbb{R})$ generated by effective divisors. By Corollary 0.3 in $[\mathbf{5 1}], X$ is uniruled.

It remains to treat the case where $\xi^{n-1} K_{X}=0$. Since $K_{X} \neq 0$, this case can't happen by the following proposition.

Proposition 2.2.2. Let $X$ be a projective variety of dimension $n$, and let $\xi_{i} \in \mathrm{NS}(X)$ be divisor classes such that

(1) $\xi_{1}$ is pseudo-effective;

(2) $\xi_{i}(i>1)$ are ample;

(3) $\prod_{i} \xi_{i}=0$.

Then $\xi_{1}=0$.

Proof. There is nothing needed to prove if $n \leq 1$. If $n=2$, we will use Hodge index theorem: since $\xi_{1} \cdot \xi_{2}=0$, one has $\xi_{1}^{2} \leq 0$, and the equality holds only when $\xi_{1}=0$. On the other hand, we may take $N$ a positive integer such that $N \xi_{2}+\xi_{1}$ is ample and thus has non-negative intersection with $\xi_{1}$ as $\xi_{1}$ is pseudo-effective. Thus we have

$$
\xi_{1}^{2}=\left(\xi_{1}+N \xi_{2}\right) \xi_{1} \geq 0 .
$$

Combining with Hodge index theorem, we have $\xi_{1}=0$.

Now we assume that $n \geq 3$ and we want to reduce to the case $n=2$ and to use the following Lefshetz Theorem in hyperplane section ([27], page 156): Let $D_{i}$ be smooth divisors $D_{i}(i=3, \ldots n)$ representing positive multiples of $\xi_{i}$ such that the partial products $Y_{k}:=\prod_{i=k+1}^{n} D_{i}$ are smooth subvarieties of $X$ of dimension $k$. Then for each $k$, the restriction map

$$
H^{2}\left(Y_{k}, \mathbb{Q}\right) \longrightarrow H^{2}\left(Y_{k-1}, \mathbb{Q}\right)
$$


is an isomorphism when $k \geq 4$, and injective when $k=3$.

By induction we can show that the restriction of $\xi_{1}$ on $Y_{k}$ is pseudoeffective. It is sufficient to show that any effective divisor $A$ of $Y_{k+1}$ will have the restriction $[A] \cdot Y_{k}$ represented by an effective divisor. Indeed, write $A=B+m D_{k}$ with $B$ properly intersecting $D_{k}$, and then $[A] \cdot Y_{k}$ will be represented by $\left[B \cdot D_{k}\right]+m\left[D_{k}^{\prime}\right]$ where $D_{k}^{\prime}$ is some effective representative of $\xi_{k}$ on $Y_{k}$, which always exists as $\xi_{k}$ is ample.

Now on the surface $Y_{2}, \xi_{1}$ and $\xi_{2}$ satisfy the conditions of the Proposition, so we must have that $\xi_{1}=0$ on $Y_{2}$. Now we apply the Lefshetz Theorem on the hyperplane section to conclude that $\xi_{1}=0$ on $X$.

REMARK 2.2.3. The above proposition can be considered as a supplement to Theorem 2.2 in $[\mathbf{5 1}]$ which says that a class $\alpha \in \mathrm{NS}(X)_{\mathbb{R}}$ is pseudoeffective if and only if it is in the dual of the cone $\operatorname{SME}(\mathrm{X})$ of strongly movable curves. Our proposition just says that the pairing of $\alpha$ on $\operatorname{SME}(\mathrm{X})$ is strictly positive if $\alpha \neq 0$.

Rationally connected factorization. Let us discuss some factorization results of Miyaoka and Mori [43] (see also Campana [12]). Let $X$ be a projective variety. Then their result says that there is a rational morphism $f: X \longrightarrow Y$ classifying the rational connected components, i.e., the following conditions hold:

(1) $f$ is dominated with rationally connected fiber;

(2) there is a Zariski open subset $X^{*}$ over which $f$ is regular and proper;

(3) for a general point $x$ of $X$, the fiber of $f$ over $x$ is the set $R(x)$ of points $y$ which can be connected to $x$ by a finite chain of rational curves. Here "general" means outside of a countably many proper subvarieties.

We may pick up a canonical $f: X \longrightarrow Y$ as follows: let $Y$ be the Zariski closure of points $[R(x)]$ in the Chow variety $\mathcal{C}(X)$ corresponding to the general points of $X$. Let

$$
(\rho, \pi): \widetilde{X} \longrightarrow X \times Y
$$

the universal family of cycles parameterized by $Y$. Then the morphism $\rho: \widetilde{X} \longrightarrow X$ is birational. We define $f=\pi \circ i^{-1}$ as a rational morphism

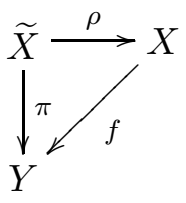

If $X$ has an endomorphism $\phi: X \longrightarrow X$ with polarization by $\phi$, then $\phi$ takes rational curves to rational curves, and thus takes $R(x)$ to $R(\phi(x))$. In other words, $\phi$ induces an endomorphism $\psi$ on $Y$ and an endomorphism $\widetilde{\phi}$ 
on $\widetilde{X}$ with commutative diagrams
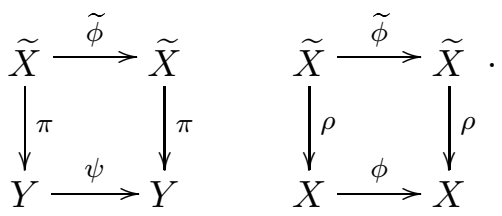

Proposition 2.2.4. Both endomorphisms $\widetilde{\phi}$ and $\psi$ are polarizable.

Proof. Let $\mathcal{M}$ Deligne's pairing on $Y$ :

$$
\mathcal{M}:=\int_{\widetilde{X} / Y} \rho^{*} \mathcal{L}^{\langle d+1\rangle}
$$

where $d$ is the relative dimension of $\pi$. Then $\mathcal{M}$ is an ample line bundle and $\psi^{*} \mathcal{M}=\mathcal{M}^{q}$. In other words $\psi$ is polarized by $\mathcal{M}$. For the polarization of $\widetilde{\phi}$, we notice that $\rho^{*} \mathcal{L}$ is ample on each fiber of $\pi$ with property

$$
\widetilde{\phi}^{*} \rho^{*} \mathcal{L}=\rho^{*} \phi^{*} \mathcal{L}=\rho^{*} \mathcal{L}^{q} .
$$

Thus for some positive number $N$,

$$
\widetilde{\mathcal{L}}:=\rho^{*} \mathcal{L} \otimes \pi^{*} \mathcal{M}^{N}
$$

will be ample with the property

$$
\widetilde{\phi^{*}} \widetilde{\mathcal{L}}=\widetilde{\mathcal{L}}^{q} .
$$

Thus we obtain a polarization for $\widetilde{\phi}$.

Remarks 2.2.5. Here are some obvious questions about the classifications of the general dynamical system:

(1) extend Proposition 2.1.1 and 2.2.1 to general Kähler variety. We may replace them by a bi-rationally equivalent dynamic system if they are helpful;

(2) classify the dynamical system into two extreme cases: the nonuniruled case, and the rationally connected case. It is not true that every rationally connected variety carries an endomorphism of degree $\geq 2$. For example, Beauville $[6]$ showed that any smooth hypersurface in projective space with dimension $\geq 2$ and degree $\geq 3$ does not admit any endomorphism of degree $\geq 2$.

REMARK 2.2.6. For algebraic endomorphism $\phi: X \longrightarrow X$ with a polarization, we are in the opposite situation of general type: $X$ and the finite étale coverings do not admit a rational map to a positive dimensional variety of general type. See Harris-Tschinkel [31] and Campana [13] for a detailed discussion of the geometry and arithmetic of these varieties of special type in contrast to varieties of general type. 
2.3. Dynamic surfaces. In the following we would like to classify the dynamic systems on surfaces.

Proposition 2.3.1. Let $\phi: X \longrightarrow X$ be an endomorphism of a Kähler surface. Then $\phi$ is polarizable if and only if $X$ is one of the following types:

(1) complex torus;

(2) hyperelliptic surfaces, i.e., the unramified quotients of the product of two genus 1 curves;

(3) toric surfaces, i.e., the completions of $\mathbb{G}_{m}^{2}$ with extending action by $\mathbb{G}_{m}^{2}$

(4) a ruled surface $\mathbb{P}_{C}(\mathcal{E})$ over an elliptic curve such that either

(a) $\mathcal{E}=\mathcal{O}_{C} \oplus \mathcal{M}$ with $\mathcal{M}$ torsion or of positive degree;

(b) $\mathcal{E}$ is not decomposable and has odd degree.

Proof. By a result of Fujimoto and Nakayama ([25], Theorem 1.1), the only non-algebraic Kähler surfaces admitting endomorphisms of degree $\geq 2$ are complex tori. So we will only consider algebraic ones. By Proposition 2.1.1, we need only consider unramified quotients of abelian surfaces and algebraic surfaces with negative Kodaira dimension. So we have the first two cases listed above, plus rational surfaces and irrational ruled surfaces.

By a result of Noboru Nakayama ([46], Theorem 3), a rational surface $X$ has an endomorphism $\phi$ of degree $\geq 2$ if and only if it is toric. We may take $\phi$ to be the "square morphism" on $X$, i.e., the morphism on $X$ satisfies the equation $\phi(t x)=t^{2} \phi(x)$ for any $t \in \mathbb{G}_{m}^{2}$ and $x \in X$. For polarization, we may simply take $\mathcal{L}$ to be the divisor of the complement of $\mathbb{G}_{m}^{2}$ in $X$ :

$$
\phi^{*} \mathcal{L}=\mathcal{L}^{2} .
$$

It remains to work on $\mathbb{P}^{1}$-bundle $\pi: X \longrightarrow C$ over a curve of genus $\neq 0$. We will use an idea of Nakayama ([46], proof of Proposition 5). We need to check when such an $X$ has a polarizable endomorphism $\phi$. Notice that any such $\phi$ will take rational curves to rational curves. Thus $\phi$ will dominate an endomorphism $g$ of $C$ :

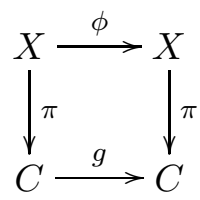

Let $g^{*} X=X \times{ }_{g} C$, then $\phi$ is the decomposition $X \stackrel{\alpha}{\longrightarrow} g^{*} X \stackrel{\beta}{\longrightarrow} X, \alpha$ is a morphism over $C$, and $\beta$ is the projection. Let $\mathcal{L}$ be an ample line bundle on $X$ such that

$$
\phi^{*} \mathcal{L} \simeq \mathcal{L}^{q}
$$

It follows that $\alpha$ has degree $q$. Since $\operatorname{deg} \phi=q^{2}$, it follows also that $\operatorname{deg} \beta=q$, and that $C$ must have genus 1 .

Lemma 2.3.2. Let $g: C \longrightarrow C$ be a morphism of curve of genus 1 of degree $q>1$. Then any endomorphism $\phi: X \longrightarrow X$ of ruled surface 
over $C$ is induced by a homomorphism $g: C \longrightarrow C$ of degree $q$, and a homomorphism of vector bundles

$$
g^{*} \mathcal{E} \longrightarrow \operatorname{Sym}^{q} \mathcal{E} \otimes \mathcal{N}
$$

with $\mathcal{N}$ a line bundle on $C$. Moreover, $\phi$ is polarized if and only if $\operatorname{deg} \mathcal{N}=0$.

Proof. The first statement is well known. It remains to study when such $\phi$ is polarizable. Let $\mathcal{L}_{0}$ be the $\mathcal{O}(1)$-bundle corresponding to $\mathcal{E}$. Then we can write

$$
\mathcal{L}=\mathcal{L}_{0}^{m} \otimes \pi^{*} \mathcal{N}_{0} .
$$

Here $\mathcal{N}_{0}$ is some line bundle on $C$. The equation $\phi^{*} \mathcal{L}=\mathcal{L}^{q}$ is equivalent to

$$
\left(\phi^{*} \mathcal{L}_{0} \otimes \mathcal{L}_{0}^{-q}\right)^{m}=\pi^{*}\left(g^{*} \mathcal{N}_{0} \otimes \mathcal{N}_{0}^{-q}\right) .
$$

This equality shows that $\phi^{*} \mathcal{L}_{0} \otimes \mathcal{L}_{0}^{-q}$ has degree 0 on all fibers. It follows that for some bundle $\mathcal{N}$ on $C$,

$$
\phi^{*} \mathcal{L}_{0} \simeq \mathcal{L}_{0}^{q} \otimes \pi^{*} \mathcal{N}, \quad g^{*} \mathcal{N}_{0} \simeq \mathcal{N}_{0}^{q} \otimes \mathcal{N}^{m} .
$$

Since $\operatorname{deg} g=q$, the second equation gives $\operatorname{deg} \mathcal{N}=0$.

Conversely, if $\phi$ is induced by a homomorphism as in the lemma with $\operatorname{deg} \mathcal{N}=0$, then we may find a line bundle $\mathcal{N}_{0}$ on $C$ of degree 1 such that $g^{*} \mathcal{N}_{0} \simeq \mathcal{N}_{0}^{q} \otimes \mathcal{N}$. Then we can check that $\mathcal{L}:=\mathcal{L}_{0} \otimes \pi^{*} \mathcal{N}_{0}$ will give the right polarization for $\phi$.

After being twisted by a line bundle on $C$, any vector bundle of rank 2 on $C$ is one of the following three types:

(1) there is a splitting, $\mathcal{E} \simeq \mathcal{O}_{C} \oplus \mathcal{M}$ with $\operatorname{deg} \mathcal{M} \geq 0$;

(2) there is a non-split exact sequence

$$
0 \longrightarrow \mathcal{O}_{C} \longrightarrow \mathcal{E} \longrightarrow \mathcal{O}_{C} \longrightarrow 0 ;
$$

(3) there is a non-split exact sequence

$$
0 \longrightarrow \mathcal{O}_{C} \longrightarrow \mathcal{E} \longrightarrow \mathcal{M} \longrightarrow 0
$$

where $\operatorname{deg} \mathcal{M}=1$.

In case (1), since $C$ has genus 1 there is a point $O$ such that $\mathcal{M}=$ $\mathcal{O}_{C}(d \cdot O)$ if $d=\operatorname{deg} \mathcal{M}>0$. We give $C$ an elliptic curve structure such that $O$ is the unit element. Let $a \geq 2$ be a fixed integer. Then the multiplication by $a$ gives

$$
[a]^{*} \mathcal{M} \simeq \mathcal{M}^{b}, \quad b= \begin{cases}a^{2}, & \text { if } \operatorname{deg} \mathcal{M} \neq 0, \\ a, & \text { if } \operatorname{deg} \mathcal{M}=0 .\end{cases}
$$

If $\mathcal{M}$ is torsion of order $t$, then we may take $a=t+1$, and we can replace $b$ by $a^{2}$. Thus in the case that either $\operatorname{deg} \mathcal{M}>0$ or $\mathcal{M}$ is torsion, we have a morphism of bundles on $C$ :

$$
[a]^{*} \mathcal{E} \simeq \mathcal{O}_{C} \oplus \mathcal{M}^{a^{2}} \longrightarrow \operatorname{Sym}^{a^{2}} \mathcal{E} .
$$

This induces a morphism $\phi: X \longrightarrow X$ such that $\phi$ is compatible with multiplication $[a]$ on $C$. By the lemma, this homomorphism has polarization. 
We want to show that when $\mathcal{M}$ is a non-torsion degree 0 bundle, any homomorphism $\phi: X \longrightarrow X$ is not polarizable. Otherwise, we will have a morphism $g: C \longrightarrow C$ of degree $q>1$ and a homomorphism of vector bundles:

$$
g^{*} \mathcal{E} \longrightarrow \operatorname{Sym}^{q} \mathcal{E} \otimes \mathcal{N}
$$

where $\mathcal{N}$ is of degree 0 . Let $x$ be the section of $\mathcal{E}$ corresponding to the embedding $1 \in \mathcal{O}_{C} \subset \mathcal{E}$. Then the above equation gives

$$
\mathcal{O}_{C} \oplus g^{*} \mathcal{M} \longrightarrow \sum_{i=0}^{q} x^{q-i} \mathcal{M}^{i} \otimes \mathcal{N}
$$

Since $\mathcal{M}$ is not torsion, the bundles in the right hand side are not isomorphic to each other. Since all of them have degree 0 , we have $i$ and $j$ such that the above homomorphism is given by two isomorphisms:

$$
\mathcal{O}_{C} \simeq \mathcal{M}^{i} \otimes \mathcal{N}, \quad g^{*} \mathcal{M}=\mathcal{M}^{j} \otimes \mathcal{N}
$$

Since this homomorphism defines morphism $X \longrightarrow X$, one must have that $\{i, j\}=\{0, q\}$. Thus in any case, we have

$$
g^{*} \mathcal{M}=\mathcal{M}^{ \pm q}
$$

Let $O$ be a fixed point of $g$. Then we may consider $C$ as an elliptic curve with origin $O$. Write $\mathcal{M}=\mathcal{O}(P-O)$. Then $g^{*} \mathcal{M}=\mathcal{O}\left(\left[g^{\vee} O\right]-[O]\right)$ and $\mathcal{M}^{q}=\mathcal{O}([q P]-O)$, where $g^{\vee}$ is the conjugate of $g: g g^{\vee}=\operatorname{deg} g=q$. Thus the above equation gives

$$
g^{\vee} P= \pm q P, \quad\left(g^{\vee} \mp q\right) P=0 .
$$

This implies again that $P$ is torsion. Thus we have a contradiction.

In case $(2)$, the bundle $g^{*} \mathcal{E}$ is still non-split so it is isomorphic to $\mathcal{E}$. In other words $g^{*} X$ is isomorphic to $X$. Indeed, the extension

$$
0 \longrightarrow \mathcal{O}_{C} \longrightarrow \mathcal{E} \longrightarrow \mathcal{O}_{C} \longrightarrow 0
$$

is given by a nonzero element $t$ in $H^{1}\left(C, \mathcal{O}_{C}\right)$. The $g^{*} \mathcal{E}$ will correspond to $g^{*} t$ in $H^{1}\left(C, \mathcal{O}_{C}\right)$. As $H^{1}\left(C, \mathcal{O}_{C}\right) \simeq k, g^{*} t=a t$ with $a \in k^{\times}$, there is a homomorphism $g^{*} \mathcal{E} \simeq \mathcal{E}$ over $C$.

Thus, $\phi$ induces (and is induced by) a $C$-endomorphism of $X$ of degree $q>1$. Now we want to apply a result of Silverman about the moduli space of endomorphisms of $\mathbb{P}^{1}[\mathbf{5 6}]$. For a positive integer $d$, let Rat $_{d}$ denote the space of endomorphisms of $\mathbb{P}^{1}$. We only take the base $\mathbb{C}$ here. Then $R_{a} t_{d}$ has a natural action by $\operatorname{Aut}\left(\mathbb{P}^{1}\right)$ as follows:

$$
(h, f) \mapsto f \circ h \circ f^{-1}, \quad h \in \operatorname{Rat}_{d}, f \in \operatorname{Aut}\left(\mathbb{P}^{1}\right) .
$$

Let $\mathrm{SL}_{2} \longrightarrow$ Aut $\left(\mathbb{P}^{1}\right)$ be the Mobius transformation. Then by Theorem 1.1 and Theorem 3.2 in [56], the quotient $M_{d}:=$ Rat $_{d} / \mathrm{SL}_{2}$ exists as an affine variety over $\mathbb{C}$. In order to apply this to our situation, we give a slightly different interpretation of $M_{d}: M_{d}$ is a fine moduli space of triples $(V, h, \ell)$ where $V$ is a vector space of dimension $2, \phi$ is an endomorphism of the projective line $\mathbb{P}(V)$, and $\ell$ is an isomorphism $\operatorname{det} V \simeq \mathbb{C}$. 
In case (2), since we have an isomorphism $\ell$ : $\operatorname{det} \mathcal{E} \simeq \mathcal{O}_{X}$, the morphism $\alpha$ induces an morphism $C \longrightarrow M_{q}$. As $C$ is projective and $M_{q}$ is projective, we must have that this morphism is constant. Thus $\mathcal{E}$ must be a trivial vector on $C$. We get a contradiction!

In case (3), we claim that $X \simeq \operatorname{Sym}^{2} C$; thus, $X$ has an endomorphism by multiplication by 2 . First there is a section $O$ so that $\mathcal{M}=\mathcal{O}_{C}(O)$. In this way, $C$ becomes an elliptic curve with origin $O$. Let us consider the following maps
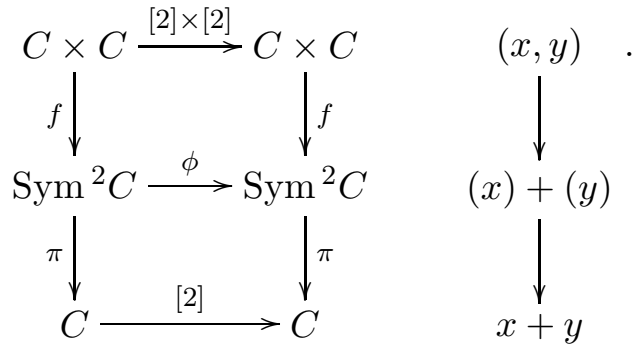

Let $p_{1}, p_{2}$ be two projections of $C \times C$ to $C$. Let $\mathcal{N}=\pi_{1}^{*} \mathcal{O}_{C}(O)+\pi_{2}^{*} \mathcal{O}(O)$ be a line bundle on $C \times C$, and $\mathcal{L}$ the descent bundle of $\mathcal{N}$ on $\mathrm{Sym}^{2} C$. The bundle $\mathcal{L}$ is ample, with fiber on $(P)+(Q)$ canonically isomorphic to $\left.\left.\mathcal{O}(O)\right|_{P} \otimes \mathcal{O}(O)\right|_{Q}$ up to the order of tensor product. The multiplication on $C \times C$ by an integer $a$ induces an endomorphism $\phi$ on $\mathrm{Sym}^{2} C$. As $[a]^{*} \mathcal{N} \simeq \mathcal{N}^{a^{2}}, \phi^{*} \mathcal{L} \simeq \mathcal{L}^{a^{2}}$

We claim the following:

(1) $\mathcal{L}$ has degree 1 on the fiber of $\pi$, thus $\mathcal{V}:=\pi_{*} \mathcal{L}$ is a rank 2 bundle on $C$;

(2) $\Gamma(\mathcal{L})=\Gamma(\mathcal{M})$ is one dimensional, say generated by $\ell$, such that $\operatorname{div}(\ell)=s^{*} C$, where $s(P)=(P)+(O)$;

(3) $s^{*} \mathcal{L} \simeq \mathcal{O}(O)$.

This claim implies that $X \simeq \mathbb{P}(\mathcal{V})$ and that $\mathcal{V}$ is not decomposable and fits in an exact sequence:

$$
0 \longrightarrow \mathcal{O}_{C} \longrightarrow \mathcal{V} \longrightarrow \mathcal{O}_{C}(O) \longrightarrow 0 \text {. }
$$

This of course implies that $\mathcal{V} \simeq \mathcal{E}$ and $X \simeq \mathrm{Sym}^{2} C$.

It remains to prove our claim. For item (1) we need to check the degree of $\mathcal{L}$ on the fiber $\operatorname{Sym}^{2}(C \times C)_{O}$ over $O \in C$. The pull-back of this fiber on $C \times C$ is the image of the following map

$$
\delta: C \longrightarrow C \times C, \quad p \mapsto(p,-p) .
$$

It follows that

$$
\begin{aligned}
\mathcal{L} \cdot \operatorname{Sym}^{2}(C)_{0} & =\frac{1}{2} \pi^{*} \mathcal{L} \cdot \pi^{*} \operatorname{Sym}^{2}(C)_{0}=\frac{1}{2} \mathcal{N} \cdot \delta(C) \\
& =\frac{1}{2} \operatorname{deg} \delta^{*}(\mathcal{N})=\frac{1}{2} \operatorname{deg} \mathcal{O}(O) \otimes[-1]^{*} \mathcal{O}(O)=1 .
\end{aligned}
$$


For item $(2)$ we see that $\Gamma(\mathcal{N})$ is equal to the symmetric part of $\Gamma(\mathcal{L})$. It is easy see that

$$
\Gamma(\mathcal{N})=p_{1}^{*} \Gamma(\mathcal{O}(O)) \otimes p_{2}^{*} \Gamma(\mathcal{O}(O))=\mathbb{C} p_{1}^{*} \alpha \otimes p_{2}^{*} \alpha
$$

where $\alpha$ is the canonical section of $\mathcal{O}(O)$ with divisor $O$. It is clear that the section $p_{1}^{*} \alpha \otimes \pi_{2}^{*} \alpha$ is symmetric and thus descends to section $\ell$ on $\mathcal{L}$ with divisor $\operatorname{div}(\ell)=s^{*} C$. For the last part, for any point $P \in C$, we see that

$$
\left.s^{*} \mathcal{L} \simeq \mathcal{O}(O)\right|_{O} \otimes \mathcal{O}(O) \simeq \mathcal{O}(O)
$$

This completes the proof of the claim.

\section{Canonical metrics and measures}

We will fix an endomorphism $\phi: X \longrightarrow X$ of a Kähler variety with a polarization by a Kähler class $\xi$. Our aim in this section is to study the distributional properties of the set Prep $(X)$ of all preperiodic points on $X$. We will first construct a canonical current $\omega$ to represent $\xi$. The class $\omega$ is integrable in the sense that the restriction of $\omega^{d}$ on any subvariety $Y$ of dimension $d$ defines a measure. By a result of Bedford-Taylor and Demailly [20], the support of the measure is Zariski dense. Then we conjecture some properties about this invariant measure. First of all, this measure can be obtained from any smooth measure by iterations. Second, this measure can be constructed from the probability measures of the backward images of a general point. We will prove some of these properties in the special cases using the work of Yau [59] and Briend-Duval [9]. Some of our results follow from some very general results of Dinh-Sibony [21], Corollary 5.4.11 and Theorem 5.4.12. We present here a self-contained treatment for the simplicity. Also our treatment is completely global and thus easily extended to $p$-adic Berkovich spaces.

Finally, with hope to initiate a dynamic Nevanlinna theory of holomorphic curves, we construct a canonical order function on a Kähler dynamical system. As an application we will show that the Fatou set is Kobayashi hyperbolic.

One question remains unsolved: the positivity of a canonical current on the Chow variety.

3.1. Canonical forms and currents. First we will try to find canonical representatives for the classes in $H^{1,1}(X)$. Let $\mathcal{Z}^{1,1}(X)$ denote the space of $\partial$ and $\bar{\partial}$ closed currents on $X$ which have the form $\omega+\frac{\partial \bar{\partial}}{\pi i} g$ with $\omega$ smooth and $g$ continuous. Then there is a class map

$$
c: \mathcal{Z}^{1,1}(X) \longrightarrow H^{1,1}(X) \text {. }
$$

Notice that $\phi^{*}$ acts on both spaces and this class map is a homomorphism of $\phi^{*}$-modules. The kernel of $c$ is the space of forms $\frac{\partial \bar{\partial}}{\pi i} g$ for continuous functions $g$. 
Proposition 3.1.1. The class map c of $\phi^{*}$-modules has a unique section, i.e., there is a unique $\phi^{*}$-subspace $\mathcal{H}^{1,1}(X)$ of $\mathcal{Z}^{1,1}(X)$ such that $c$ induces an isomorphism

$$
\mathcal{H}^{1,1}(X) \simeq H^{1,1}(X)
$$

The space $\mathcal{H}^{1,1}(X)$ is called the space of canonical forms. Moreover, if $\xi$ is an eigenclass of $\phi^{*}$ with eigenvalue $\lambda$ which is represented by a smooth form $\omega_{0}$ then the canonical lifting is the limit

$$
\omega:=\lim _{k \rightarrow \infty}\left(\lambda^{-1} \phi^{*}\right)^{k} \omega_{0} .
$$

Proof. Let $C(X)$ denote the space of continuous functions on $X$. Then we have an exact sequence

$$
0 \longrightarrow \mathbb{C} \longrightarrow C(X) \longrightarrow \mathcal{Z}^{1,1}(X) \longrightarrow H^{1,1}(X) \longrightarrow 0
$$

where the map $C(X) \longrightarrow \mathcal{Z}^{1,1}(X)$ is given by $\frac{\partial \bar{\partial}}{\pi i}$. Let $P(T)$ be the characteristic polynomial of $\phi^{*}$ acting on $H^{1,1}(X)$. We want to show that for $P\left(\phi^{*}\right)$ is invertible over $C(X)$. In this way, we may take $\mathcal{H}^{1,1}(X)=\operatorname{ker} P\left(\phi^{*}\right)$. In other words, every element $\kappa$ in $H^{1,1}(X)$ has a lifting $\eta$ such that $P\left(\phi^{*}\right) \eta=0$. Indeed, if $\eta_{0}$ is one lifting of $\kappa$ in $\mathcal{Z}^{1,1}(X)$ then $P\left(\phi^{*}\right) \eta_{0}$ is in the image of $C(X)$. Thus we have a $g \in C(X)$ such that

$$
P\left(\phi^{*}\right) \eta_{0}=\frac{\partial \bar{\partial}}{\pi i} g
$$

It is easy to see that $\kappa$ has a lifting in the kernel of $P\left(\phi^{*}\right)$ with the following form:

$$
\eta=\eta_{0}+\frac{\partial \bar{\partial}}{\pi i} P\left(\phi^{*}\right)^{-1} g
$$

It remains to show that $P\left(\phi^{*}\right)$ is invertible over $C(X)$. We write $P(T)=$ $\prod_{i}\left(T-\lambda_{i}\right)$ where $\lambda_{i}$ are eigenvalues of $\phi^{*}$ on $H^{1,1}(X)$. By Theorem 1.1.2, all $\left|\lambda_{i}\right|>1$. It follows that $\lambda_{i}^{-1} \phi^{*}$ is a compact operator on $C(X)$. Indeed,

$$
\left\|\lambda_{i}^{-1} \phi^{*} \alpha\right\|_{\text {sup }} \leq\left|\lambda_{i}\right|^{-1}\|\alpha\|_{\text {sup }}, \quad \alpha \in C(X) .
$$

It follows that $P\left(\phi^{*}\right)$ has an inverse on $C(X)$ :

$$
P\left(\phi^{*}\right)^{-1} \alpha:=\prod_{i} \sum_{k}-\lambda_{i}\left(\lambda_{i}^{-1} \phi^{*}\right)^{k} \alpha .
$$

This proves the first part of the proposition.

For the second part, let $g$ be a smooth function such that

$$
\left(1-\lambda^{-1} \phi^{*}\right) \omega_{0}+\frac{\partial \bar{\partial}}{\pi i} g=0 .
$$

Then

$$
\left.\left(\lambda^{-1} \phi^{*}\right)^{j} \omega_{0}-\left(\lambda^{-1} \phi^{*}\right)^{j+1}\right) \omega_{0}+\frac{\partial \bar{\partial}}{\pi i}\left(\lambda^{-1} \phi^{*}\right)^{j} g=0
$$


Add the above equality from $j=1$ to $j=k-1$ to obtain

$$
\left(\lambda^{-1} \phi^{*}\right)^{k} \omega_{0}=\omega_{0}+\frac{\partial \bar{\partial}}{\pi i} \sum_{j=1}^{k}\left(\lambda^{-1} \phi^{*}\right)^{j} g .
$$

It is easy to see from this expression that $\left(\lambda^{-1} \phi^{*}\right)^{k} \omega_{0}$ has a limit as the canonical lifting of $\xi$ :

$$
\omega=\omega_{0}+\frac{\partial \bar{\partial}}{\pi i}\left(1-\lambda \phi^{*}\right)^{-1} g
$$

We have an analogue for algebraic polarizations. Let $\phi: X \longrightarrow X$ be an endomorphism with a polarization. Let $\widehat{\operatorname{Pic}}(X)$ denote the group of (continuously) metrized line bundles on $X$. Then we have a class map

$$
\gamma: \widehat{\operatorname{Pic}}(X) \longrightarrow \operatorname{Pic}(X) \text {. }
$$

Again $\phi^{*}$ acts on both groups and this map is a homomorphism of $\phi^{*}$ modules.

Proposition 3.1.2. The class map $\gamma$ has a projective section, i.e., there is a unique $\phi^{*}$-submodule $\overline{\mathrm{Pic}}(X)$ of $\widehat{\mathrm{Pic}}(X)$ such that the map $\gamma$ induces an exact sequence

$$
0 \longrightarrow \mathbb{R} \longrightarrow \overline{\operatorname{Pic}}(X) \longrightarrow \operatorname{Pic}(X) \longrightarrow 0 .
$$

Here $\mathbb{R}$ maps $r \in \mathbb{R}$ to the metrized line bundle $\left(\mathcal{O}_{X},\|1\|=e^{-r}\right)$. The metrics in $\overline{\mathrm{Pic}}(X)$ are called canonical metrics.

Proof. By Proposition 3.1.1, for any line bundle $\mathcal{L}$ there is a unique metric up to a constant with curvature in $\mathcal{H}^{1,1}(X)$.

REMARKS 3.1.3. The proof of the above proposition applies to $H^{p, p}(X)$ and Green's currents for codimension $p$-cycles if we can show that $\lambda^{-1} \phi^{*}$ is compact on the space $C^{p-1, p-1}(X)$ of continuous $(p, p)$-forms on $X$. For example, if $\phi$ is polarized by a Kähler class $\xi$ which has a lifting $\omega$ which is continuous and positive pointwise, then we may equip $C^{p-1, p-1}(X)$ with norm by $\omega$. In this way we have

$$
\left\|\phi^{*} \alpha\right\|(x)=q^{p-1}\|\alpha\|(\phi(x)), \quad \alpha \in C^{p, p}(X) .
$$

Then by Theorem 1.1.2, the eigenvalue $\lambda$ on $H^{p, p}(X, \mathbb{C})$ has absolute value $q^{p}$ and again

$$
\left\|\lambda^{-1} \phi^{*} \alpha\right\|_{\text {sup }} \leq q^{-1}\|\alpha\|_{\text {sup }}
$$

In the following we want to study the volume forms defined by polarizations by $\xi$. Let $\omega$ denote its canonical form in $\mathcal{H}^{1,1}(X)$. If $\omega$ is a continuous form then we will have a volume form

$$
d \mu_{Y}=\left.\omega\right|_{Y} ^{\operatorname{dim} Y} / \operatorname{vol}(Y)
$$


Here

$$
\operatorname{vol}(Y)=\xi^{\operatorname{dim} Y} \cdot[Y]=\int_{Y} \omega_{0}^{\operatorname{dim} Y} .
$$

Only require $\omega$ is to be a current, and the above definition does not make sense. In the following we will use the limit process to show that the above definition still gives a measure. Let's study a slightly more general situation.

Let $d=\operatorname{dim} Y$ and pick up $d$ classes $\eta_{1}, \ldots, \eta_{d}$ so that

(1) $\eta_{i}$ are semi-positive;

(2) $\phi^{*} \eta_{i}=\lambda_{i} \eta_{i}$ with $\lambda>1$.

Let $\omega_{i 0}$ be semipositive, smooth forms for $\eta_{i}$. Let $\omega_{i k}=\lambda_{i}^{-k}\left(\phi^{*}\right)^{k} \omega_{i 0}$.

Proposition 3.1.4. With notation as above the following hold:

(1) $\omega_{i k}$ converges to the canonical lifting $\omega_{k}$ of $\eta_{k}$ as a current,

(2) the volume form $\omega_{1 k} \cdots \omega_{d k} \delta_{Y}$ is weakly convergent with a limit measure

$$
\omega_{1} \cdots \omega_{d} \delta_{Y}:=\lim _{k \rightarrow \infty} \omega_{1 k} \cdots \omega_{d k} \delta_{Y}
$$

on $Y$ which is independent of the choice of initial forms $\omega_{i 0}$.

Integrable forms and metrics. We want to show that the proposition follows a more general theory about integrable metrics [62]. More precisely, a class $\omega=\omega_{0}+\frac{\partial \bar{\partial}}{\pi i} g \in \mathcal{Z}^{1,1}$ is called semi-positive if $g=\lim _{n} g_{n}$ is the limit in $C(X)$ of a sequence of smooth functions $g_{n}$ such that $\omega_{0}+\frac{\partial \bar{\partial}}{\pi i} g_{n}$ are smooth positive forms. A class $\omega$ is called integrable if $\omega$ is the difference $\omega_{1}-\omega_{2}$ of two semi-positive classes. Let $\mathcal{S}^{1,1}(X)$ denote the space of integrable forms. A function $g$ is called a Green's function if there is a divisor $D=\sum_{i} a_{i} D_{i}$ on $X$ with real coefficients such that $g$ is continuous on $X \backslash D$ with logarithmic singularity near $D$ : if locally $D_{i}$ is defined by equations $f_{i}=0$ near a point $x$, then $g$ has an asymptotic formula near $x$ :

$$
g=\sum a_{i} \log \left|f_{i}\right|+h
$$

where $h$ is a continuous function. Let $\mathcal{G}(X)$ denote the space of Green's functions.

Proposition 3.1.5. Let $X$ be a compact Kähler variety and let $Y$ be a subvariety of dimension $d$. There is a unique integration pairing

$$
\begin{gathered}
\mathcal{G}(Y) \times \mathcal{S}^{1,1}(X)^{d} \longrightarrow \mathbb{C}, \\
\left(g, \omega_{1}, \ldots, \omega_{d}\right) \mapsto \int_{Y} g \omega_{1} \cdots \omega_{d}
\end{gathered}
$$

such that the following properties are verified:

(1) the pairing is linear in each variable;

(2) if each $\omega_{i}$ is semi-positive and is a limit of smooth forms $\omega_{i k}$ on $X$, then the above pairing is the limit of the usual integral pairings of smooth forms. 
Proof. Write $\omega_{i}=\lim \omega_{i k}$ with $\omega_{i k}$ smooth and positive. Thus we have $\omega_{i k}=\omega_{i 0}+\frac{\partial \bar{\partial}}{\pi i} h_{i k}$ with $h_{i k}$ smooth and convergent to $h_{i}$. First we want to show that the functional

$$
g \mapsto \int_{Y} g \omega_{1 k} \cdots \omega_{d k}
$$

is convergent on the restriction of $g \in \mathcal{G}^{\infty}(X)$ on $Y$, the space of functions whose local asymptotic formula (3.1.3) has smooth $h$. Let $g$ be a smooth function on $X$; the difference of the integrations is given by

$$
\begin{aligned}
& \int_{Y} g\left(\omega_{1 k} \cdots \omega_{d k}-\omega_{1 \ell} \cdots \omega_{d \ell}\right) \\
& =\int_{Y} g \sum_{i=1}^{d} \omega_{1 k} \cdots \omega_{i-1, k}\left(\omega_{i, k}-\omega_{i, \ell}\right) \omega_{i+1, \ell} \cdots \omega_{d, \ell} .
\end{aligned}
$$

From our expression of $\omega_{i k}$,

$$
\omega_{i k}-\omega_{i j}=\frac{\partial \bar{\partial}}{\pi i}\left(h_{i k}-h_{i \ell}\right) .
$$

It follows that

$$
\begin{aligned}
& \int_{Y} g \cdot\left(\omega_{1 k} \cdots \omega_{d k}-\omega_{1 \ell} \cdots \omega_{d \ell}\right) \\
& =\int_{Y} \sum_{i=1}^{d} \omega_{1 k} \cdots \omega_{i-1, k}\left(h_{i, k}-h_{i, \ell}\right) \omega_{i+1, \ell} \cdots \omega_{d, \ell} \frac{\partial \bar{\partial}}{\pi i} g .
\end{aligned}
$$

Since $g$ is smooth, we have a formula

$$
\frac{\partial \bar{\partial}}{\pi i} g=\sum a_{i} \delta_{D_{i}}-\alpha
$$

where $\alpha$ is a smooth $(1,1)$-form. Let $M$ be a positive number such that

$$
\omega_{i, 0}^{\prime}:=\omega_{i 0}-\frac{1}{M} \alpha
$$

is positive point wise. Then the above sum can be written as

$$
\begin{aligned}
& \int_{Y} g \cdot\left(\omega_{1 k} \cdots \omega_{d k}-\omega_{1 \ell} \cdots \omega_{d \ell}\right) \\
& =\sum_{j} a_{j} \int_{D_{j}} \sum_{i=1}^{d} \omega_{1 k} \cdots \omega_{i-1, k}\left(h_{i, k}-h_{i, \ell}\right) \\
& \quad+M \int_{Y} \sum_{i=1}^{d} \omega_{1 k} \cdots \omega_{i-1, k}\left(h_{i, k}-h_{i, \ell}\right) \omega_{i+1, \ell} \cdots \omega_{d, \ell}\left(\omega_{i 0}^{\prime}-\omega_{i 0}\right) .
\end{aligned}
$$


Replacing $h_{i, k}-h_{i, \ell}$ by its $L^{\infty}$-norm and $\omega_{i 0}^{\prime}-\omega_{i 0}$ by $\omega_{i 0}^{\prime}+\omega_{i 0}$, we have the following estimate:

$$
\begin{aligned}
& \left|\int_{Y} g \cdot\left(\omega_{1 k} \cdots \omega_{d k}-\omega_{1 \ell} \cdots \omega_{d \ell}\right)\right| \\
& \leq \sum_{i}\left(\sum_{j}\left|a_{j}\right|\left(\eta_{1} \cdots \widehat{\eta}_{i} \cdots \eta_{d}\left[D_{i}\right]\right)+2 M\left(\eta_{1} \cdots \eta_{d}[Y]\right)\right)\left\|h_{i k}-h_{i \ell}\right\|_{\text {sup }},
\end{aligned}
$$

where $\eta_{i}$ are the classes of $\omega_{i}$ in $H^{1,1}(X, \mathbb{R})$. This shows that $\omega_{1 k} \cdots \omega_{d k} \delta_{Y}$ converges as a distribution, say $\omega_{1} \cdots \omega_{d} \delta_{Y}$. To show this limit can be extended into a continuous Green's function, we need only consider the continuous function $g \in C(X)$, or equivalently show that the limits is actually a measure. It suffices to show the following:

(1) the functional on $C^{\infty}(X), g \longrightarrow \int_{Y} g \omega_{1} \cdots \omega_{d}$ is continuous with respect to the supreme norm and

(2) the restriction of $C^{\infty}(X)$ on $Y$ is dense in $C(Y)$.

The first property is clear since $\omega_{1 k} \cdots \omega_{1, d}$ is semi-positive with volume $\left.\eta_{1} \cdots \eta_{d}\right|_{Y}$. For a smooth function $f$ on $X$ :

$$
\left|\int_{Y} g \cdot \omega_{1} \cdots \omega_{d}\right|=\lim _{k}\left|\int_{Y} g \omega_{1 k} \cdots \omega_{d k}\right| \leq\|g\|_{\text {sup }}\left(\eta_{1} \cdots \eta_{d}[Y]\right) .
$$

For the second property, we use Stone-Weierstrass theorem: $C^{\infty}(X)$ is dense in $C(X)$ which is surjective on $C(Y)$ by restriction map.

Finally, we want to show the independence on $\omega_{i k}$. This can be done by the same argument as above. Indeed, let $\omega_{i k}^{\prime}$ be different smooth and positive forms convergent to $\omega_{i}$, which induce a differential sequence of forms $\omega_{1 k}^{\prime} \cdots \omega_{d k}^{\prime}$. The same argument as above can be used to show that

$$
\left|\int_{Y} g \cdot\left(\omega_{1 k}^{\prime} \cdots \omega_{d k}^{\prime}-\omega_{1 k} \cdots \omega_{d k}\right)\right| \leq C \sum_{i}\left\|\alpha_{i k}\right\|_{\sup }
$$

where $C$ is a constant depending only on $f$, and $\alpha_{i k}$ are smooth functions such that

$$
\omega_{i k}^{\prime}-\omega_{i k}=\frac{\partial \bar{\partial}}{\pi i} \alpha_{i k}
$$

It is easy to show that $\alpha_{i k} \longrightarrow 0$. Thus two limits are same.

For any open connected subset (in complex topology) $U$ of a subvariety $Y$ of $X$ of dimension $d$ and the current $\omega_{1} \cdots \omega_{d}$ defined by integrable forms $\omega_{1}, \ldots, \omega_{d}$, the support of $\omega_{1} \cdots \omega_{d}$ is defined as the smallest closed subset $\operatorname{Supp}_{U}\left(\omega_{1} \cdots \omega_{d}\right)$ of $Y$ in complex topology such that

$$
\int_{Y} f \omega_{1} \cdots \omega_{d}=0
$$

whenever $f \in C_{0}(U)$ vanishes on $\operatorname{Supp}_{U}\left(\omega_{1} \cdots \omega_{d}\right)$. When $X$ is projective, the above proposition shows that $\operatorname{Supp}_{Y}\left(\omega_{1} \cdots \omega_{d}\right)$ is not included in any 
proper subvariety. Otherwise, $\operatorname{Supp}_{Y}\left(\omega_{1} \cdots \omega_{d}\right)$ will be included in the support of an effective divisor $D$. Then we can take $g$ to be the Green's function for $D$. The integral will be infinite! This contradicts our proposition.

For general Kähler variety, Chambert-Loir pointed to the following result of Bedford-Taylor and Demailly:

THEOREM 3.1.6 (Bedford-Taylor-Demailly).

The set $\operatorname{Supp}_{U}\left(\omega_{1} \cdots \omega_{d}\right)$ is either empty or Zariski dense in $U$.

Proof. When $Y$ is smooth, this is simply a result of Bedford-Taylor and Demailly [20], Corollary 2.3. In the general case, let $\pi: \widetilde{Y} \longrightarrow Y$ be a resolution of singularity. Then we can define the pull-back of forms in $\mathcal{Z}^{1,1}$ by the usual way: if $\omega=\omega_{0}+\frac{\partial \bar{\partial}}{\pi i} g$ then

$$
\pi^{*} \omega=\pi^{*} \omega_{0}+\frac{\partial \bar{\partial}}{\pi i} g \circ \pi .
$$

If $\omega$ is integrable, then it is easy to show that $\pi^{*} \omega$ is integrable. For any continuous function $f$ on $Y$, and any integrable currents $\omega_{1}, \ldots, \omega_{d}$, it is easy to check that

$$
\int_{\widetilde{Y}} \pi^{*} f \cdot \pi^{*} \omega_{1} \cdots \pi^{*} \omega_{d}=\int_{Y} f \cdot \omega_{1} \cdots \omega_{d} .
$$

It follows that

$$
\pi^{-1} \operatorname{Supp}_{U}\left(\omega_{1} \cdots \omega_{d}\right) \subset \operatorname{Supp}_{\pi^{-1} U}\left(\pi^{*} \omega_{1} \cdots \pi^{*} \omega_{d}\right) .
$$

Thus we are reduced to the smooth case.

Remark 3.1.7. The same proof as in Proposition 3.1.5 can be used to show the following weaker form of Theorem 3.1.6: Assume that $X$ is projective; then the measure $\left.\omega_{1} \cdots \omega_{d}\right|_{Y}$ on $Y$ does not support on any proper subvariety. Let $D$ be a any divisor of $Y$, and $g$ be a Green's function for $D$, i.e., a function on $Y$ with logarithmic singularity such that

$$
\frac{\partial \bar{\partial}}{\pi i} g=\delta_{D}-h
$$

where $h$ is a smooth $(1,1)$ form on $Y$. We need to show that the integral

$$
\int_{Y} \omega_{1} \cdots \omega_{d}
$$

makes sense and is finite, which then implies that the support of the measure is not supported on $D$.

Metrics on Chow varieties. In the following we want to introduce the canonical forms or metrics for the Chow varieties and show their compatibility with the induced endomorphism $\phi_{*}$ and $\phi_{d, \delta, k}$. One basic question in this theory is about the fiber pairing of integrable metrics on $X$. 
Question 3.1.8. Let $X$ be a compact Kähler variety. How could one construct an integration pairing

$$
\begin{gathered}
\mathcal{S}^{1,1}(X)^{d+1} \longrightarrow \mathcal{S}^{1,1}\left(\mathcal{C}_{d}(X)\right), \\
\left(\omega_{0}, \ldots, \omega_{d}\right) \mapsto \int_{\mathcal{Z}_{d}(X) / \mathcal{C}_{d}(X)} \omega_{0} \cdots \omega_{d} ?
\end{gathered}
$$

When $X$ is algebraic, this question has a positive answer, see [63]. Mimicking what has been done in the projective case, our first step to answer this question is to restrict to smooth forms $\omega_{i}^{\prime}$ in the same class of $\omega_{i}$ and try to show that the above integral defines some integral forms. If $\omega_{i}=\omega_{i}^{\prime}+\frac{\partial \bar{\partial}}{\pi i} h_{i}$ then we compute the difference formally by:

$$
\begin{aligned}
& \int_{\mathcal{Z}_{d}(X) / \mathcal{C}_{d}(X)} \omega_{0} \cdots \omega_{d}-\int_{\mathcal{Z}_{d}(X) / \mathcal{C}_{d}(X)} \omega_{0} \cdots \omega_{d} \\
& =\int_{\mathcal{Z}_{d}(X) / \mathcal{C}_{d}(X)} \sum_{i=0}^{d} \omega_{1} \cdots \omega_{i-1}\left(\omega_{i}-\omega_{i}^{\prime}\right) \omega_{i+1}^{\prime} \cdots \omega_{d}^{\prime} \\
& =\int_{\mathcal{Z}_{d}(X) / \mathcal{C}_{d}(X)} \sum_{i=0}^{d} \omega_{1} \cdots \omega_{i-1} \frac{\partial \bar{\partial}}{\pi i} h_{i} \omega_{i+1}^{\prime} \cdots \omega_{d}^{\prime} \\
& =\frac{\partial \bar{\partial}}{\pi i} \int_{\mathcal{Z}_{d}(X) / \mathcal{C}_{d}(X)} h_{i} \sum_{i=0}^{d} \omega_{1} \cdots \omega_{i-1} \omega_{i+1}^{\prime} \cdots \omega_{d}^{\prime} .
\end{aligned}
$$

Our Proposition 3.1.5 shows the last integral is well defined at each point. But then one has to prove that this integral defines a continuous function on $\mathcal{C}_{d}(X)$.

Let $\phi: X \longrightarrow X$ be an endomorphism of a compact Kähler variety with a polarization by a positive class $\xi$ which is represented by a canonical form $\omega$. If Question 3.1.8 has a positive answer, then we will have canonical forms $\omega_{d}$ on the varieties $\mathcal{C}_{d}(X)$ compatible with the action of $\phi^{*}$. Of course for the variety $\mathcal{C}(d, \delta, k)$ which is of finite type, we will have the usual theory of canonical metrics.

3.2. Equidistribution of backward orbits. Fix an endomorphism $\phi: X \longrightarrow X$ of a compact Kähler variety with polarization. Let $d \mu_{0}$ be a continuous probability measure. Define $d \mu_{k}$ by the following inductive formula

$$
d \mu_{k}=\frac{\phi^{*} d \mu_{k-1}}{\operatorname{deg} \phi}
$$

More precisely, for any continuous function $f$ on $X$,

$$
\int_{X} f d \mu_{k} d \mu_{k}=(\operatorname{deg} \phi)^{-k} \int_{X} \phi_{*}^{k} f d \mu_{0} .
$$


Here $\phi_{*}^{k} f$ is a function defined by

$$
\phi_{*}^{k} f(x)=\sum_{\phi^{k}(y)=x} f(y)
$$

where the sum is over pre-images of $x$ with multiplicity. Notice that $\phi_{*}^{k}(f)$ is a bounded on $X$ and continuous on a Zariski open subset of $X$, and thus is measurable with respect to any continuous measure. The following is a simple consequence of a result of Yau:

THEOREM 3.2.1. Let $\phi: X \longrightarrow X$ be an endomorphism of a compact Kähler manifold with polarization $\xi$. Then $d \mu_{k}$ converges to the canonical measure on $X$ :

$$
\lim _{k \rightarrow \infty} d \mu_{k}=\omega^{n} /\left(\xi^{n}[X]\right)
$$

where $\omega$ is the canonical form for the class $\xi$ and $n=\operatorname{dim} X$.

Proof. Notice that for a continuous function $f$ on $X$,

$$
\int f d \mu_{k}=(\operatorname{deg} \phi)^{-k} \int \phi_{*}^{k}(f) d \mu_{0}
$$

where $\phi_{*}^{k}(f)$ is defined such that

$$
\phi_{*}^{k}(f)(x)=\sum_{\phi^{k}(y)=x} f(x)
$$

where the sum is over the preimage of $x$ under $\phi^{k}$ with multiplicity. It is easy to check that $\phi_{*}^{k}(f)$ is bounded and continuous on a Zariski open subset of $X$. So the above integral makes sense.

As every continuous measure is a strong limit of smooth volume forms, we may assume that $d \mu_{0}$ is a smooth volume form. By a theorem of Yau [59], $d \mu_{0}$ on $X$ is induced from a unique class $\omega_{0}$ in $\xi$ by formula

$$
d \mu_{0}=\omega_{0}^{n} /\left(\xi^{n}[X]\right) .
$$

Now we can apply Proposition 3.1.4.

We would like to conjecture that this theorem is true without assumption on smoothness of $X$ :

Conjecture 3.2.2. Let $\phi: X \longrightarrow X$ be an endomorphism of a (possibly singular) compact Kähler variety with a polarization $\xi$. Let $d \mu_{0}$ be a continuous probability measure on $X$. Define $d \mu_{k}$ by inductive formula

$$
d \mu_{k}=\frac{\phi^{*} d \mu_{k-1}}{\operatorname{deg} \phi} .
$$

Then $d \mu_{k}$ is convergent to the probability measure $d \mu_{X}$ of the form

$$
\omega^{n} /\left(\xi^{n}[X]\right), \quad(n=\operatorname{dim} X .)
$$


Let $\phi: X \longrightarrow X$ be an endomorphism of a compact Kähler variety with a polarization $\xi$. Let $x$ be a point in $X$. For any positive integer $k$, let's define a probability measure the $k$-th preimage:

$$
\mu_{x, k}=(\operatorname{deg} \phi)^{-k} \sum_{\phi^{k}(y)=x} \delta_{y}
$$

where the sum is over the $k$-th preimage with multiplicity.

By this conjecture, for almost all $p$ the $\mu_{p, n}$ is convergent to the canonical measure $d \mu$. Indeed, for any continuous probability measure $d \mu_{0}$, the $d \mu_{n}$ in Theorem 3.1.6 can be written as

$$
\int_{X} f d \mu_{n}=\int_{X}\left\{\int_{X} f(x) d \mu_{p, n}(x)\right\} d \mu(p) .
$$

In a more precise way we would like to make the following conjecture:

Conjecture 3.2.3. Let $x$ be a point of $X$ and let $Y$ be the Zariski closure of the complete orbit, i.e., $Y$ is the minimal subvariety of $X$ containing all $\phi^{-k}(x)$ and $\phi^{k}(x)$ for all $k$. Then $\mu_{p, n}$ is weakly convergent to the canonical measure $\omega^{\operatorname{dim} Y} / \operatorname{vol}(Y)$ on $Y$, where $\omega^{\operatorname{dim} Y}$ is the canonical measure on $Y$ defined by the canonical form $\omega$ on $Y$.

It seems that a more natural subvariety than $Y$ for the conjecture is the backward limit of $x$ : i.e., the minimal subvariety $Y^{\prime}$ containing $\phi^{-k}(x)$ for $k$ sufficiently large. We claim that $Y=Y^{\prime}$. Indeed, for each $k \geq 0$ let $Y_{k}$ be the Zariski closure of the union $\cup_{i \geq k} \phi^{-k}(x)$. Then $Y_{k}$ is a decreasing sequence of closed subvarieties of $X$. Thus $Y=Y_{k}=\cap Y_{k}$ for $k$ sufficiently large. It is easy to see that $Y$ is periodic, so $Y=Y^{\prime}$.

When $X=\mathbb{P}^{N}$, the conjecture is a theorem by recent work of BriendDuval [9] and Briend-Cantat-Shishikura [10]. Indeed by Briend-CantatShishikura, the full orbit of any $x \in \mathbb{P}^{N}$ is always a finite union of linear subspace, and thus isomorphic to $\mathbb{P}^{k}$ if $\phi$ is replaced by a power. Then by Briend-Duval $\mu_{m, x}$ is equidistributed on $\mathbb{P}^{k}$.

3.3. Hyperbolicity and holomorphic curves. Let $\phi: X \longrightarrow X$ be an endomorphism of a compact Kähler variety with a polarization. As discussed in $\S 2.3, X$ should have a fibration over a variety $Y$ of Kodaira dimension 0 with rationally connected fibers. Thus $X$ should be special in the sense of Campana [13]. By Campana, Conjecture 9.2 in [13], this should be equivalent to hyperbolically special:

Conjecture 3.3.1. Let $\phi: X \longrightarrow X$ be an endomorphism of a compact Kähler variety with a polarization. Then the pseudo-metric $d_{X}$ on $X$ vanishes.

Here the pseudo-metric is defined in Kobayashi's hyperbolic geometry [34]. We can only prove the vanishing of the pseudo-distance under Conjecture 3.2.3: 
Proposition 3.3.2. Let $\phi: X \longrightarrow X$ be an endomorphism of a compact Kähler variety with a polarization. Assume Conjecture 3.2.1 for the endomorphism $\phi \times \phi$ for $X \times X$. Then the pseudo-distance vanishes on $X$.

Proof. For two positive number $a>0$, let $T(a)$ be the open subset of $X \times X$ of points $(x, y)$ with the pseudo-distance satisfying the inequality $d(x, y)>a$. Since the pseudo-distance is decreasing under $\phi$ :

$$
d(\phi x, \phi y) \leq d(x, y)
$$

we see that $\phi^{-1} T(a) \subset T(a)$.

If $T(a)$ is not empty, then it has a non-empty interior, and thus supports continuous probability measure $d \mu_{0}$. By our assumption, the limit $\operatorname{deg} \phi^{-k} \phi^{* k} d \mu_{0}$ converges to the canonical measure on $X \times X$. Notice that the canonical measure $d \mu_{X} \times d \mu_{X}$ on $X \times X$ is the product measure on $X$ 's. Thus the support of this measure contains the support of $d \mu_{X}$ by diagonal map $X \rightarrow X \times X$. It follows that $T(a)$ contains the diagonal elements. This is a contradiction as the distance of $d(x, x)=0$ for any $x \in X$. So we have shown that $d(x, y)=0$ for all $x, y \in X$.

Combined with Theorem 3.2.1, we can prove the conjecture for endomorphisms with polarizations:

Corollary 3.3.3. Let $\phi: X \longrightarrow X$ be an endomorphism of a compact Kähler manifold with a polarization by a Kähler form. Then the pseudodistance vanishes on $X$ everywhere.

One consequence of Conjecture 3.3.1 is the vanishing pseudo-volume form of Kobayashi which is apparently easy to prove:

Proposition 3.3.4. The Kobayashi pseudo-measure vanishes.

Proof. Let $\Phi_{X}$ denote the Kobayashi pseudo-volume form. Then $\phi^{*} \Phi_{X}$ $\leq \Phi_{X}$. Taking integration of both sides we obtain

$$
\operatorname{deg} \phi \int \Phi \leq \int \Phi
$$

It follows that $\int \Phi=0$ or $\Phi=0$ almost everywhere.

Another consequence is the existence of many non-degenerate holomorphic maps $\mathbb{C} \longrightarrow X$ by work of Brody (see Lang [36], Theorem 2.2). Fix a Kähler form $\omega_{0}$ on $X$ which induces a metric on $X$. From the proof of Theorem 2.2. in [36], one sees that for any point $x \in X$ and any tangent vector $v \in T_{X, x}$ of norm 1 , there is a holomorphic map $f: \mathbb{C} \longrightarrow X$ such that

$$
d f_{0}(\partial / \partial z)=v, \quad\left\|d f_{z}\right\| \leq 1, \quad \forall z \in \mathbb{C} .
$$

Here $d f_{z}$ is the map $T_{\mathbb{C}, z} \longrightarrow T_{X, f(x)}$ between two tangent spaces with norms induced by the Euclidean norm on $\mathbb{C}$ and the norm $\omega_{0}$ on $X$. 
Holomorphic curves. Fix a Kähler dynamical system $(X, \phi, \xi)$. Let $\omega$ be the canonical form. The remainder of this section is devoted to proving the following:

THEOREM 3.3.5. Let $C$ be an affine complex curve; i.e., the complement of finitely many points in a Riemann surface $\bar{C}$. There is no nonconstant holomorphic map $\psi: C \longrightarrow X$ such that $\psi^{*}(\omega)=0$.

REMARK 3.3.6. Let $F$ be the complement of the support of $\omega$. As in Sibony [54] Theorem 1.6.5, one may show that $F$ is the Fatou set of $\phi$, i.e., the set of points $p$ which has neighborhood $U$ such that $\left.f\right|_{U}$ is equicontinuous. Thus our theorem shows that $F$ is Brody hyperbolic, which is equivalent to Kobayashi hyberbolic as $F$ is the complement of a compact set.

Our main idea for the proof is to use Nevanlinna theory of holomorphic curves. We refer to Griffiths $[\mathbf{2 8}]$ for basic theory. Let's fix a holomorphic map $p: \bar{C} \longrightarrow \mathbb{P}^{1}$ such that $p^{-1} \mathbb{C} \subset C$. Replacing $C$ by $p^{-1} \mathbb{C}$ we may simply assume that $C$ has a finite and flat morphism to the affine line $\mathbb{C}$.

Order function. For each positive number $r$, let $C_{r}$ denote the set of points $p \in C$ such that $|p(z)| \leq r$. Let $\omega_{0}$ be a closed $(1,1)$ form in the class. Then we can define the order function (or characteristic function) for $C_{r}$ as follows:

$$
T_{\omega_{0}}\left(C_{r}\right)=\int_{0}^{r} \frac{d t}{t} \int_{C_{t}} \psi^{*} \omega_{0}
$$

If $\omega_{0}^{\prime}$ is in the same class as $\omega_{0}$, i.e., there is a real function $g \in C^{\infty}(X)$ such that $\omega_{0}^{\prime}=\omega_{0}+\frac{\partial \bar{\partial}}{\pi i} g$, then

$$
\begin{aligned}
T_{\omega_{0}^{\prime}}\left(C_{r}\right)-T_{\omega_{0}}\left(C_{r}\right) & =\int_{0}^{r} \frac{d t}{t} \int_{C_{t}} \frac{\partial \bar{\partial}}{\pi i} \psi^{*} g \\
& =\frac{1}{\pi i} \int_{0}^{r} \frac{d t}{t} \int_{\partial C_{t}} \bar{\partial}\left(\psi^{*} g\right)=\frac{1}{\pi i} \int_{0}^{r} \frac{d t}{t} \int_{\partial \mathbb{C}_{t}} \bar{\partial}\left(p_{*} \psi^{*} g\right) .
\end{aligned}
$$

Now we use polar coordinate $z=t e^{i \theta}, \bar{z}=t e^{-i \theta}$, and

$$
\bar{\partial}=\frac{\partial}{\partial \bar{z}} \otimes d \bar{z}=\frac{1}{2}\left(\frac{\partial}{\partial t}+\frac{i}{t} \frac{\partial}{\partial \theta}\right) \otimes(d t-i t d \theta) .
$$

It follows that

$$
\begin{aligned}
T_{\omega_{0}^{\prime}}\left(C_{t}\right)-T_{\omega_{0}}\left(C_{t}\right) & =\frac{-1}{2 \pi} \int_{0}^{r} d t \int_{0}^{2 \pi}\left(\frac{\partial}{\partial t}+\frac{i}{t} \frac{\partial}{\partial \theta}\right) p_{*} \psi^{*} g d \theta \\
& =\frac{-1}{2 \pi} \int_{0}^{r} d t \int_{0}^{2 \pi} \frac{\partial}{\partial t} p_{*} \psi^{*} g d \theta \\
& =\frac{-1}{2 \pi} \int_{0}^{2 \pi}\left(p_{*} \psi^{*} g(t, \theta)-p_{*} \psi^{*} g(0, \theta)\right) d \theta
\end{aligned}
$$

In summary we have the formula:

$$
T_{\omega_{0}^{\prime}}\left(C_{r}\right)-T_{\omega_{0}}\left(C_{r}\right)=\frac{-1}{2 \pi} \int_{\partial C_{r}} \psi^{*} g p^{*} d \theta+\psi^{*} g\left(p^{*} 0\right)
$$


In terms of supreme norm, we have

$$
\left|T_{\omega_{0}^{\prime}}\left(C_{r}\right)-T_{\omega_{0}}\left(C_{r}\right)\right| \leq 2 \operatorname{deg} p \cdot\|g\|_{\text {sup }}
$$

First Nevanlinna Inequality. Notice that every $\omega_{0}$ is induced from a metric $\|\cdot\|_{0}$ on the line bundle $\mathcal{L}$ on $X$. Let $s$ be a nonzero rational section of $\mathcal{L}$. Assume that $D=\operatorname{div} s$ intersects $C$ properly and $\psi p^{-1}(0)$ is disjoint with $D$. Then

$$
\omega=\delta_{D}+\frac{\partial \bar{\partial}}{\pi i} \log \|s\|_{0}
$$

where $\delta_{D}$ is the Dirac distribution of $D$, and $\frac{\partial \bar{\partial}}{\pi i}$ is computed in the sense of distributions. The same formal computation gives

$$
\begin{aligned}
T_{\omega_{0}}\left(C_{r}\right) & =\frac{1}{\pi} \int_{0}^{t} \frac{d t}{t} \int_{C_{r}}\left(\delta_{D}+\frac{\partial \bar{\partial}}{\pi i} \log \|s\|_{0}\right) \\
& =\frac{1}{\pi} \int_{0}^{r}\left(C_{t} \cdot D\right) \frac{d t}{t}-\int_{\partial C_{r}} \psi^{*} \log \|s\|_{0} \frac{d \theta}{2 \pi}+\log \|s\|_{0}\left(p^{*} 0\right) .
\end{aligned}
$$

Define the counting function and proximity function by

$$
N_{D}\left(C_{r}\right)=\frac{1}{\pi} \int_{0}^{r}\left(C_{t} \cdot \psi^{*} D\right) \frac{d t}{t}
$$

and

$$
m_{s}\left(\partial C_{t}\right)=-\int_{\partial C_{r}} \psi^{*} \log \|s\|_{0} \frac{d \theta}{2 \pi}+\log \|s\|_{0}\left(p^{*} 0\right) .
$$

Then the identity we just proved is the First Main Theorem in Nevanlina theory:

$$
T_{\omega_{0}}\left(C_{r}\right)=N_{D}\left(C_{r}\right)+m_{s}\left(\partial C_{r}\right) .
$$

If $s$ is regular, then last two terms are bounded from below. Thus we obtain the Nevanlina inequality:

$$
N_{D}\left(C_{r}\right) \leq T_{\omega}\left(C_{r}\right)+O(1) .
$$

One consequence of this inequality is that $C$ is algebraic if

$$
T_{\omega}\left(C_{r}\right)=O(\log r) .
$$

Indeed, if this equality holds, then by (3.3.3), $C \cdot f^{*} D$ will be bounded by some positive number $e$. It follows that $C$ is an algebraic curve of degree at most $e$. The converse is also true, see Griffiths [28]. In fact, assume that $\mathcal{L}$ is ample on the Zariski closure $Y$ of $f(C)$, and let $Y \longrightarrow \mathbb{P}^{m}$ be the embedding defined by $\mathcal{L}$. Then with respect to a Fubini-Study metric on $\mathbb{P}^{m}$ and its dual metric on $\mathbb{P}^{m *}$, there is a Crofton formula ([28], pp. 22-23):

$$
\int_{\mathbb{P}^{m *}} N_{D}\left(C_{r}\right) d D=T_{\omega_{0}}\left(C_{r}\right)
$$


Proof of Theorem 3.3.5. Assume $\omega_{0}$ is semi-positive and apply this to $\omega^{\prime}=d^{-k} \phi^{*} \omega_{0}$. Then we can define the order function for the canonical form $\omega$. Write $\omega=\omega_{0}+\frac{\partial \bar{\partial}}{\pi i} g$. As $\psi^{*} \omega=0$, the above inequality implies the following

$$
\left|T_{\omega_{0}}\left(C_{r}\right)\right| \leq 2 \operatorname{deg} p\|g\|_{\text {sup }} .
$$

Thus the order function of $\psi$ is finite. It follows that $\psi$ can be extended to a holomorphic map $\bar{\phi}: \bar{C} \longrightarrow X$. In this case $\omega$ will be supported on a finite number of points. This is impossible as there is no continuous function $g$ such that $\frac{\partial \bar{\partial}}{\pi i} g$ gives a Dirac measure on points, see Theorem 3.1.6.

\section{Arithmetic dynamics}

In this section we will study (polarized) endomorphisms defined over a number field. We will first propose a conjecture about the equidistribution of the Galois orbits of a dynamically generic sequence of preperiodic points. This equidistribution conjecture implies the dynamic Manin-Mumford conjecture assuming the Zariski density conjecture of support of canonical measures. Then we will define the canonical heights and generalize these conjectures to small points. Following Chambert-Loir, we also propose an equidistrubution conjecture on Berkovich's p-adic analytic spaces. Finally, we will prove an equidistribution theorem for Zariski generic sequences of small points on any variety using the recent work of Yuan on arithmetic bigness. One immediate consequence is the equivalence of the dynamic Bogomolov conjecture and the dynamic equidistrubution conjecture.

4.1. Preperiodic points and small points. Let $\phi: X \longrightarrow X$ be an endomorphism of a projective variety with a polarization defined over a number field $K$. In other words, this means that $X$ and $\phi$ are defined over $K$, and that there is an ample line bundle $\mathcal{L} \in \operatorname{Pic}(X) \otimes \mathbb{R}$ such that $\phi^{*} \mathcal{L}=\mathcal{L}^{q}$ for some integer $q>1$.

The set Prep $(X)$ of preperiodic points is defined over $\bar{K}$. Moreover, by a theorem of Northcott [47], for each number $D$, the set of preperiodic points $x$ with degree $\operatorname{deg}(x):=[K(x): K] \leq D$ is finite. Let $\Gamma$ denote the absolute Galois group over $K: \Gamma=\operatorname{Gal}(\bar{K} / K)$. Then $\Gamma$ acts on the set Prep $(X)$ of preperiodic points on $X$.

Let us fix an embedding $\bar{K} \subset \mathbb{C}$ and write $X(\mathbb{C})$ for complex points via this embedding. Let $d \mu$ be the probability measure on $X(\mathbb{C})$ defined by the Chern class $c_{1}$ of the bundle $\mathcal{L}_{\mathbb{C}}$ constructed in $\S 3.1 .1$. Notice that $d \mu$ is the invariant measure on $X$; i.e., the probability measure $d \mu$ defined on $X$ such that

$$
\phi^{*} d \mu=\operatorname{deg} \phi \cdot d \mu .
$$

Conjecture 4.1.1 (Equidistribution of dynamically generic preperiodic points). Let $x_{i}$ be a sequence of preperiodic points on $X$ such that no infinite subsequence is supported in a proper preperiodic subvariety. Then the 
Galois orbits of $x_{i}$ are equidistributed with respect to the canonical measure $d \mu$ on $X(\mathbb{C})$.

More precisely, for $x \in \operatorname{Prep}(X)$, define probability measure

$$
\mu_{\Gamma x}:=\frac{1}{\operatorname{deg} x} \sum_{y \in \Gamma x} \delta_{y} .
$$

Then the conjecture says the probability measures $\delta_{\Gamma x_{n}}$ converge weakly to the invariant measure $d \mu$ in the following sense: for a continuous function $f$ on $X(\mathbb{C})$,

$$
\frac{1}{\operatorname{deg}\left(x_{n}\right)} \sum_{y \in \Gamma x_{n}} f(y) \longrightarrow \int_{X(\mathbb{C})} f(x) d \mu(x)
$$

as $n \longrightarrow \infty$.

Consequences. In the following let us give some consequences of the conjecture. The first consequence is the dynamic Manin-Mumford Conjecture 1.2.1.

Proposition 4.1.2. Conjecture 4.1.1 $\Rightarrow$ Conjecture 1.2.1.

Proof. Let $Y$ be a subvariety containing a Zariski dense subset of preperiodic points $\operatorname{Prep}(X) \cap Y$ of $\phi$. There is nothing to prove if $\operatorname{dim} Y=0$. Otherwise Prep $(X) \cap Y$ is an infinite set. Let $Z$ be the intersection of all preperiodic subvarieties defined over $K$ of $X$ containing $Y$. Then $Z$ itself is preperiodic. As the set of all algebraic subvarieties of $X$ is countable, we may list the set of preperiodic proper subvarieties of $Z$ in a sequence $Z_{1}, \ldots, Z_{n}, \cdots$. Using induction, we can define a sequence of points $x_{n} \in \operatorname{Prep}(X) \cap Y$ such that $x_{n} \notin \cup_{i=1}^{n} Z_{i}$. In this way, the sequence $x_{n}$ will have finite intersection with any proper preperiodic subvariety of $Z$. Now we apply Conjecture 4.1.1 to conclude that the Galois orbits of $x_{n}$ converge to the invariant measure on $Z$. As all Galois orbits lie in $Y$, the invariant measure $d \mu$ must be supported on $Y$. Now by Theorem 3.1.6, $Y=Z$. In other words, $Y$ is preperiodic.

Topological interpretation. To understand the nature of the equidistribution conjecture, let's introduce the following topologies:

(1) Zariski topology: the topology defined by algebraic equations;

(2) dynamic topology: the topology defined by preperiodic subvarieties;

(3) archimedean topology: the topology defined by inequalities of usual continuous functions on $X(\mathbb{C})$;

(4) distributional topology: Let $\operatorname{Meas}(X(\mathbb{C}))$ denote the set of probability measures on $X(\mathbb{C})$ with weak topology; let's define a map

$$
\mathbb{D}: X \longrightarrow \operatorname{Meas}(X(\mathbb{C})), \quad y \mapsto d \mu_{\bar{y}(\mathbb{C})}
$$

Here $y \in X$ is a point of scheme $X$, and $\bar{y}$ denotes the Zariski closure. Then the distributional topology is the induced topology on $X$ from $\mathbb{D}$. Theorem 3.1.6 implies that the map $\mathbb{D}$ is injective. 
We have the following order of fineness of the topologies:

Distributional $>$ Archimedean $>$ Zariski $>$ Dynamic.

On Prep $(X)$, Conjecture 4.1.1 says that the dynamic topology equals the distributional topology while Conjecture 1.2.1 says that the Zariski topology equals the dynamic topology.

What is proved?

(1) If $X$ is an abelian variety, and $\phi=[m]$ is the multiplication by a positive number $m>1$, the conjecture was proved in [64] and [58].

(2) If $X=\mathbb{P}^{n}$ and $\phi$ is the morphism $\left(x_{0}, \ldots, x_{m}\right) \longrightarrow\left(x_{0}^{m}, \ldots, x_{n}^{m}\right)$, the conjecture was proved by Bilu $[8]$.

(3) If $X$ is a compactification of an almost split semi-abelian variety, then the conjecture was proved by Chambert-Loir [17].

Height machinery. In the following, we want to introduce the concept of heights and state a conjecture about small points.

Let $K$ be a number field. For each place $v$ of $K$ let $|\cdot|_{v}$ be the normalized places: for any haar measure $d x_{v}$ on $K_{v}$,

$$
d(a x)_{v}=|a|_{v} d x_{v}
$$

For a point $x=\left(x_{0}, \ldots, x_{n}\right) \in \mathbb{P}^{n}(\overline{\mathbb{Q}})$, the naive height $h_{\text {naive }}(x)$ of $x$ is defined by

$$
h_{\text {naive }}(x)=\frac{1}{[K: \mathbb{Q}]} \sum_{v} \log \max \left(\left|x_{0}\right|_{v}, \ldots,\left|x_{n}\right|_{v}\right) .
$$

It can be shown that the definition $h(x)$ can be extended to $\mathbb{P}^{n}(\overline{\mathbb{Q}})$. If $x$ is a rational point represented by an $(n+1)$-tuple of integers $\left(x_{0}, \ldots, x_{n}\right)$ with no common divisor, then

$$
h_{\text {naive }}(x)=\log \max \left(\left|x_{0}\right|_{\infty}, \ldots,\left|x_{n}\right|_{\infty}\right) .
$$

If we define the complexity $c(x)$ of $x$ as the maximum number of digits of $x_{i}$, which measures the time spent to write a number down, then

$$
h_{\text {naive }}(x)-c(x) \log 10
$$

is bounded on the set of rational points of $\mathbb{P}^{n}$. A basic property of $h_{\text {naive }}$ is the following Northcott Theorem: for any given numbers $D$ and $H$, the set of points in $\mathbb{P}^{n}$ with height $\leq H$ and degree $\leq D$ is finite.

Let $X$ be a projective variety over a number field. Let $\mathcal{L}$ be a very ample line bundle. Then there is an embedding $i: X \rightarrow \mathbb{P}_{K}^{N}$ such that $i^{*} \mathcal{O}(1) \simeq \mathcal{L}$. In this way we obtain a height function

$$
h_{\mathcal{L}}: \quad X(\overline{\mathbb{Q}}) \longrightarrow \mathbb{R}, \quad h_{\mathcal{L}}(x)=h_{\text {naive }}(i(x)) .
$$

Here are some standard properties of $h_{\mathcal{L}}$ :

(1) $h_{\mathcal{L}}$ up to a bounded function on $X(\overline{\mathbb{Q}})$ does not depend on the choice of the embedding. 
(2) $h_{\mathcal{L}}$ is additive in the sense that for two very ample line bundles $\mathcal{L}$ and $\mathcal{M}$,

$$
h_{\mathcal{L} \otimes \mathcal{M}}=h_{\mathcal{L}}+h_{\mathcal{M}}+O(1) .
$$

(3) $h_{\mathcal{L}}$ is bounded from below:

$$
h_{\mathcal{L}}(x) \geq C
$$

(4) (Northcott Theorem) for any positive numbers $D, H$, the set

$$
\left\{x \in X(\overline{\mathbb{Q}}), \quad \operatorname{deg}(x)<D, \quad h_{\mathcal{L}}(x)<H\right\}
$$

is finite.

(5) For two very ample line bundles $\mathcal{L}$ and $\mathcal{M}$, the equality $h_{\mathcal{L}}=$ $h_{\mathcal{M}}+O(1)$ implies that $\mathcal{L} \otimes \mathcal{M}^{-1}$ is a torsion line bundle.

From these properties, we can extend the definition of height function to every $\mathcal{L} \in \operatorname{Pic}(X) \otimes \mathbb{R}$ by linearity. In other words, we have an embedding from the $\mathbb{R}$-vector space of $\mathbb{R}$-bundles into the space of functions on $X(\overline{\mathbb{Q}})$ modulo bounded functions:

$$
\operatorname{Pic}(X) \otimes \mathbb{R} \longrightarrow \operatorname{Funct}(X(\overline{\mathbb{Q}}), \mathbb{R}) / O(1) .
$$

This is a homomorphism of $\mathbb{R}$-vector spaces and such that the Northcott theorem is true if $\mathcal{L}$ is numerically positive in $\operatorname{Pic}(X) \otimes \mathbb{R}$. Indeed, if $\mathcal{M} \in$ $\operatorname{Pic}(X)$ is a very ample line bundle in the usual sense, then there is a positive number $\epsilon$ such that $\mathcal{L}-\epsilon \mathcal{M}$ is positive. Thus the Northcott Theorem for $\mathcal{M}$ implies that for $\mathcal{L}$.

Now let us go back to our situation: let $\phi: X \longrightarrow X$ be an endomorphism of a projective variety over a number field $K$ with a polarization by an $\mathbb{R}$-line bundle $\mathcal{L} \in \operatorname{Pic}(X) \otimes \mathbb{R}$. Fix a height function $h_{\mathcal{L}}$. Then we can define the canonical height $\widehat{h}_{\mathcal{L}}$ on $X(\overline{\mathbb{Q}})$ by the following formula:

$$
\widehat{h}_{\mathcal{L}}(x):=\lim _{k \longrightarrow \infty} \frac{h_{\mathcal{L}}\left(\phi^{k}(x)\right)}{q^{k}} .
$$

The height $\widehat{h}_{\mathcal{L}}$ defined in this way does not depend on the choice of height function $h_{\mathcal{L}}$ : one may characterize $\widehat{h}_{\mathcal{L}}$ as the unique height function for $\mathcal{L}$ such that

$$
\widehat{h}_{\mathcal{L}}(\phi(x))=q \widehat{h}_{\mathcal{L}}(x)
$$

Then $\widehat{h}_{\mathcal{L}}$ has the following properties:

Proposition 4.1.3. For any $x \in X(\overline{\mathbb{Q}}), \widehat{h}_{\mathcal{L}}(x) \geq 0$. Moreover, $\widehat{h}_{\mathcal{L}}(x)=$ 0 if and only if $x$ is a torsion point.

Proof. Since each $\mathcal{L}$ is ample, we may choose an initial height function $h_{\mathcal{L}}$ with positive values. In this way, the above definition formula 4.1.1 implies that $\widehat{h}_{\mathcal{L}} \geq 0$. If $\widehat{h}_{\mathcal{L}}(x)=0$, then

$$
\widehat{h}_{\mathcal{L}}\left(\phi^{k}(x)\right)=0 \quad \forall k .
$$

In other words, every point in the forward orbit of $x$ has height 0 . By Northcott's theorem, this orbit must be finite. Thus $x$ must be preperiodic. 
Conversely, if $x$ is preperiodic, say, $\phi^{k}(x)=\phi^{\ell}(x)$ with $k \neq \ell$, then $\widehat{h}_{\mathcal{L}}\left(\phi^{k}(x)\right)=\widehat{h}_{\mathcal{L}}\left(\phi^{\ell}(X)\right)$, or $q^{k} \widehat{h}_{\mathcal{L}}(x)=q^{\ell} h_{\mathcal{L}}(x)$. Thus $\widehat{h}_{\mathcal{L}}(x)=0$.

Now we apply the Northcott theorem to give:

COROLlary 4.1.4. For any number D, the set of preperiodic points on $X$ with degree bounded by $D$ is finite.

REMARK 4.1.5. There are some conjectures concerning the lengths of orbits of preperiodic points and heights of non-torsion points:

(1) If $x$ is non-preperiodic then a Lehmer type conjecture states that

$$
\widehat{h}_{\mathcal{L}}(x) \geq c \cdot \operatorname{deg}(x)^{-1} .
$$

(2) If $x$ is a preperiodic point, then the length ord $(x)$ of the orbit of $x$ should have the bound

$$
\text { ord }(x) \leq c \operatorname{deg}(x) .
$$

(3) (Morton-Silverman [45]) For all positive integers $D, N, d$ with $d>$ 2 , there exists an integer $\kappa(D, N, d)$ such that for each number field of degree $D$ over $\mathbb{Q}$, and each finite endomorphism $\psi: \mathbb{P}_{k}^{N} \longrightarrow \mathbb{P}_{k}^{N}$ of degree $d^{N}$, the number of preperiodic points $\psi$ in $\mathbb{P}^{N}(k)$ is less than or equal to $\kappa(D, N, d)$. By Fakhruddin [23], this conjecture implies the corresponding uniform on torsion points on abelian varieties.

See Fakhruddin [23] for some other interesting questions about rational points on a dynamical system.

Rational points. From the classification in $\S 2$, one sees that for a polarized endomorphism $\phi: X \longrightarrow X, X$ is either a quotient of an abelian variety or a rationally connected variety. Thus it is conjectured that for some finite extension $F$ of $K$, the set of rational points $X(F)$ is Zariski dense in $X$. Here we would like to conjecture that this is already true for some orbits:

Conjecture 4.1.6. Let $\phi: X \longrightarrow X$ be an endomorphism of a projective variety defined over a number field $K$ with a polarization. Then there is a point $x \in X(\bar{K})$ such that the forward orbit

$$
\left\{x, \phi(x), \ldots, \phi^{k}(x), \ldots\right\}
$$

is Zariski dense in $X$.

Small points. In the following we want to extend the dynamic ManinMumford Conjecture 1.2.1 and the equidistribution conjecture for small points to

Conjecture 4.1.7 (Dynamic Bogomolov conjecture). A subvariety $Y$ of $X$ is preperiodic if and only if for any Zariski open subvariety $U$ of $Y$, and for any positive number $\epsilon>0$, there is a point $x \in U(\overline{\mathbb{Q}})$ such that $\widehat{h}_{\mathcal{L}}(x)<\epsilon$. 
ConjeCture 4.1.8 (Equidistribution of dynamically generic small points). Fix a complex place of $K$. Let $x_{i}$ be a sequence of points on $X$ such that no infinite subsequence is included in a proper preperiodic subvariety, and that $\lim _{i \rightarrow \infty} \widehat{h}_{\mathcal{L}}\left(x_{i}\right)=0$. Then the Galois orbits of $x_{i}$ are equidistributed with respect to the canonical measure on $X$.

What is proved?

(1) If $X$ is an abelian variety, then both conjectures were proved in $[64]$ and $[58]$.

(2) If $X$ is the multiplicative group then Conjecture 4.1.7 was proved in [61], and the equidistribution Conjecture 4.1.8 was proved by Bilu [8].

(3) If $X$ is an almost split semiabelian group, then the Bogomolov conjecture was proved by Chambert-Loir $[\mathbf{1 7}]$.

4.2. Metrized line bundles and heights of subvarieties. In this subsection, we want to associate to every metrized line bundle a height function. By an integral and hermitian model of $(X, \mathcal{L})$ we mean a triple $\bar{L}:=\left(\widetilde{X}, \widetilde{\mathcal{L}},\|\cdot\|_{v}\right)$ where

(1) $\widetilde{X}$ is an projective and flat scheme over $\operatorname{Spec} \mathcal{O}_{K}$ with generic fiber $X$

(2) $\widetilde{\mathcal{L}}$ is a line bundle on $\widetilde{X}$;

(3) $\|\cdot\|_{v}$ is a collection of metrics of $\mathcal{L}_{v}:=\mathcal{L}_{K, v} \otimes \mathbb{C}$ on $X_{v}=X \otimes_{v} \mathbb{C}$ for each embedding $v: K \longrightarrow \mathbb{C}$. We assume that for an embedding $v$ and its complex conjugate $v^{c}$,

$$
\|\ell\|_{v}(x)=\left\|\ell^{c}\right\|_{v^{c}}\left(x^{c}\right) .
$$

Here the bar map is the complex conjugation

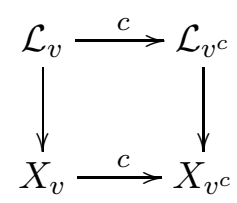

Let $x$ be a point in $X(\overline{\mathbb{Q}})$. Then the Zariski closure $\bar{x}$ of $x$ has a normalization $f: \operatorname{Spec} \mathcal{O}_{F} \rightarrow \widetilde{X}$ where $\mathcal{O}_{F}$ is the ring of integers of some number field $F$ over $K$. The invertible $\mathcal{O}_{F}$-module $\mathcal{N}:=f^{*} \mathcal{L}$ is equipped with a hermitian metric on $\mathcal{N} \otimes_{\sigma} \mathbb{C}$ for each embedding $\sigma: F \rightarrow \mathbb{C}$. Then we define the degree of $\overline{\mathcal{L}}$ on $\bar{x}$ by

$$
\operatorname{deg}_{\overline{\mathcal{L}}} \bar{x}=\log \frac{\# \mathcal{N} / n \mathcal{O}_{F}}{\prod_{v \mid \infty}\|n\|_{v}}
$$

where $n$ is any nonzero element in $\mathcal{N}$. One can show that

$$
h_{\overline{\mathcal{L}}}(x)=\frac{1}{[F: \mathbb{Q}]} \operatorname{deg}_{\overline{\mathcal{L}}}(\bar{x}) .
$$


One immediate advantage of using metrized line bundles is to extend the definition of heights to arbitrary cycles of $\widetilde{X}$ by using the intersection theory of Gillet and Soulé [26]. Indeed, on a subvariety $Y$ of $X$ over $K$ of dimension $d$, the intersection number of the metrized line bundles $\overline{\mathcal{L}}_{1}, \ldots, \overline{\mathcal{L}}_{d}$ can be computed by induction:

$$
\begin{aligned}
\widehat{c}_{1}\left(\overline{\mathcal{L}}_{1}\right) \cdots \widehat{c}_{1}\left(\overline{\mathcal{L}}_{d}\right) \cdot Y= & \widehat{c}_{1}\left(\overline{\mathcal{L}}_{1}\right) \cdots \widehat{c}_{1}\left(\overline{\mathcal{L}}_{d-1}\right) \cdot[\operatorname{div} s] \\
& -\sum_{v \mid \infty} \int_{X_{v}(\mathbb{C})} \log \|s\|_{v} c_{1}\left(\overline{\mathcal{L}}_{1, v}\right) \cdots c_{1}\left(\overline{\mathcal{L}}_{d-1, v}\right),
\end{aligned}
$$

where $s$ is a nonzero section of $\mathcal{L}_{d}$ on $Y$.

If $Y$ is a closed subvariety of $X$, then the height $h_{\overline{\mathcal{L}}}(Y)$ is defined by the formula

$$
h_{\overline{\mathcal{L}}}(Y)=\frac{\operatorname{deg}_{\overline{\mathcal{L}}}(\tilde{Y})}{(\operatorname{dim} Y+1) \operatorname{deg}_{\mathcal{L}}(Y)}
$$

where $\tilde{Y}$ is the Zariski closure of $Y$ in $\tilde{X}$.

Integrable metrized line bundles [62]. Consider a projective variety $X$ over Spec $K$. For a line bundle $\mathcal{L}$ on $X$ and an integral model $(\widetilde{X}, \widetilde{\mathcal{L}})$ of $\left(X, \mathcal{L}^{e}\right)$ over $\operatorname{Spec} \mathcal{O}_{K}$, one can define an adelic metric $\|\cdot\|_{\tilde{\mathcal{L}}}=\left\{\|\cdot\|_{v}, v \in \mathcal{S}\right\}$ on $\mathcal{L}$, where $e$ is a positive integer, $\mathcal{S}$ is the set of places of $K$, and $\|\cdot\|_{v}$ is a metric on $\mathcal{L} \otimes_{K} \bar{K}_{v}$ on $X\left(\bar{K}_{v}\right)$. The metrics constructed this way are called smooth metrics. The metric so obtained will be invariant under the Galois $\operatorname{group} \operatorname{Gal}\left(\bar{K}_{v} / K_{v}\right)$.

Let $\mathcal{L}_{1}, \ldots, \mathcal{L}_{d}(d=\operatorname{dim} X+1)$ be line bundles on $X$. For each positive integer $n$, let $\left(\widetilde{X}_{n}, \widetilde{\mathcal{L}}_{1, n}, \ldots, \widetilde{\mathcal{L}}_{d, n}\right)$ be an arithmetic model of $\left(X, \mathcal{L}_{1}^{e_{1, n}}, \ldots\right.$, $\left.\mathcal{L}_{d}^{e_{d, n}}\right)$ on $\operatorname{Spec} \mathcal{O}_{K}$. Assume for each $i$ that $\left(\mathcal{L},\|\cdot\|_{\widetilde{\mathcal{L}}_{i, n}}\right)$ converges to an adelic metrized line bundle $\overline{\mathcal{L}}_{i}$. One might ask whether the number

$$
c_{n}=\frac{c_{1}\left(\widetilde{\mathcal{L}}_{1, n}\right) \cdots c_{1}\left(\widetilde{\mathcal{L}}_{d, n}\right)}{e_{1, n} \cdots e_{d, n}}
$$

in Gillet-Soulé's intersection theory converges or not.

We showed in [62] that $c_{n}$ converges if all $\widetilde{\mathcal{L}}_{i, n}$ are relatively semipositive, and that $\lim _{n \rightarrow \infty} c_{n}$ depends only on $\overline{\mathcal{L}}_{i}$. We say that an adelic line bundle $\overline{\mathcal{L}}$ is integrable if $\overline{\mathcal{L}} \stackrel{\sim}{\rightarrow} \overline{\mathcal{L}}_{1} \otimes \overline{\mathcal{L}}_{2}^{-1}$ with $\overline{\mathcal{L}}_{i}$ semipositive. It follows that GilletSoulé's theory can be extended to integrable metrized line bundles. Some theorems such as the Hilbert-Samuel formula, the Nakai-Moishezon theorem, and the successive minima inequality remain valid on semi-smooth metrized line bundles.

Admissible metrized line bundles [62]. Let $\phi: X \rightarrow X$ be an endomorphism of a projective variety with a polarization over $K$. Thus we have an ample line bundle and an isomorphism $\alpha: \mathcal{L}^{q} \simeq \phi^{*} \mathcal{L}$ with $q>1$. Using Tate's argument, in [62] we constructed a unique integrable metric $\|\cdot\|$ on $\mathcal{L}$ such that

$$
\|\cdot\|^{q}=\alpha^{*} \phi^{*}\|\cdot\|
$$


Now for any effective cycle $Y$ of $X$ of pure dimension, we can define an (absolute) height

$$
h_{\mathcal{L}}(Y)=\frac{c_{1}\left(\left.\overline{\mathcal{L}}\right|_{Y}\right)^{\operatorname{dim} Y+1}}{(\operatorname{dim} Y+1) \operatorname{deg}_{\mathcal{L}}(Y)} .
$$

The height $h_{\mathcal{L}}$ can be characterized by the property that

$$
h_{\mathcal{L}}(\phi(Y))=q h_{\mathcal{L}}(Y) .
$$

As Tate did, $h_{\mathcal{L}}$ can be defined without an admissible metric. Some situations are studied by Philippon [49], Kramer [35], Call and Silverman [11], and Gubler $[\mathbf{2 9}]$. In this case, if $Y$ is preperiodic: the orbit

$$
\left\{Y, f(Y), f^{2}(Y), \ldots\right\}
$$

is finite, then $h_{\mathcal{L}}(Y)=0$.

We showed in $[62]$ that the Bogomolov conjecture is equivalent to the following converse:

Conjecture 4.2.1. Let $\phi: X \longrightarrow X$ be an endomorphism of a projective variety over a number field $K$ with a polarization by an ample line bundle $\mathcal{L}$. Then $h_{\mathcal{L}}(Y)=0$ if and only if $Y$ is preperiodic.

This is a theorem [61] for the case of multiplicative group. A consequence is the generalized Lang's conjecture which claims that if $Y$ is not preperiodic then the set of preperiodic points in $Y$ is not Zariski dense. Lang's conjecture is proved by Laurent [37] and by Raynaud [52] for abelian varieties.

Measures on Berkovich spaces [30] [16]. Fix a place $v$ of $K$. Then there is a $v$-adic analytic space $X_{v}^{\text {an }}-$ Berkovich space [7]. If $v$ is complex this is usual $X_{v}(\mathbb{C})$; if $v$ is real this is $X_{v}(\mathbb{C}) /\{1, c\}$ where $c$ is the complex conjugation on $X_{v}(\mathbb{C})$. For $v$ a finite place, we have an embedding of topology space $\left|X_{v}\right| \longrightarrow X_{v}^{\text {an }}$ with dense image, where $\left|X_{v}\right|$ denotes the set of closed points on $X_{v}$ with $v$-adic topology, or equivalently, the set of Galois orbits of $X\left(\bar{K}_{v}\right)$ under $\mathrm{Gal}\left(\bar{K}_{v} / K_{v}\right)$. Moreover the metrized line bundles on some model of $X_{v}$ over $\mathcal{O}_{K_{v}}$ will induce some continuously metrized line bundles on $X_{v}^{\text {an }}$ whose restriction on $\left|X_{v}\right|$ is the usual metrized line bundles constructed as above. Thus we will have the notion of integrable metrized line bundles.

A continuous function $f$ on $X_{v}^{\text {an }}$ is called smooth if its restriction on $\left|X_{v}\right|$ is the logarithm of a smooth metric $\|\cdot\|_{v}$ at $v$ of $\mathcal{O}_{X_{v}^{\text {an }}}$ defined by an integral model:

$$
f=\log \|1\| \text {. }
$$

By the work of Gubler [30], the smooth functions are dense in the continuous functions on $X_{v}^{\text {an }}$. In other words, let $C^{\infty}\left(\left|X_{v}\right|\right)$ denote the space of smooth functions on $\left|X_{v}\right|$ which may not be closed under multiplication, and let $R\left(X_{v}\right)$ be the ring of functions on $\left|X_{v}\right|$ generated by smooth functions with supremum norm. Then $C^{\infty}\left(\left|X_{v}\right|\right)$ is dense in $R\left(X_{v}\right)$ and $X^{\text {an }} v$ as a topological space is the spectrum of $R\left(X_{v}\right)$ :

$$
X_{v}^{\text {an }}=\operatorname{Hom}_{\text {cont }}\left(R\left(X_{v}\right), \mathbb{R}\right) .
$$


Here the right hand side stands for continuous homomorphisms of $\mathbb{R}$-algebras. In other words, $X$ is the unique compact space such that $C\left(X_{v}^{\text {an }}\right)=$ $R\left(\left|X_{v}\right|\right)$.

Now we consider the situation of a polarized dynamical system $(X, \phi, \mathcal{L})$. For any subvariety $Y$ of $X_{v}$ of dimension $d$ and integrable metrized line bundles $\overline{\mathcal{L}}_{1}, \ldots, \overline{\mathcal{L}}_{d}$, Chambert-Loir [16] defined the measure

$$
c_{1}\left(\overline{\mathcal{L}}_{1}\right) \cdots c_{1}\left(\overline{\mathcal{L}}_{d}\right) \delta_{Y_{v}^{\text {an }}}
$$

supported on the image $Y^{\text {an }}{ }_{v}$ which has the usual properties as in the archimedean case in $\S 3.1$

For example, for a subvariety $Y$ of $X$ over $K$ of dimension $d$ and adelic metrized line bundles $\overline{\mathcal{L}}_{1}, \ldots, \overline{\mathcal{L}}_{d}$, one can compute the intersection by induction:

$$
\begin{aligned}
\widehat{c}_{1}\left(\overline{\mathcal{L}}_{1}\right) \cdots \widehat{c}_{1}\left(\overline{\mathcal{L}}_{d}\right) \cdot Y= & \widehat{c}_{1}\left(\overline{\mathcal{L}}_{1}\right) \cdots \widehat{c}_{1}\left(\overline{\mathcal{L}}_{d-1}\right) \cdot[\operatorname{div} s] \\
& -\sum_{v} \int_{X_{v}^{\text {an }}} \log \|s\|_{v} c_{1}\left(\overline{\mathcal{L}}_{1, v}\right) \cdots c_{1}\left(\mathcal{L}_{d-1, v}\right) \delta_{Y_{v}^{\text {an }}}
\end{aligned}
$$

where $s$ is a nonzero section of $\mathcal{L}_{d}$ on $Y$.

In this case, the construction of Chambert-Loir as above gives the canonical measures on $X_{v}^{\text {an }}$ for each integral subvariety $\delta_{Y}$ for each embedding $v: K \longrightarrow \mathbb{C}_{p}$ :

$$
d \mu_{Y, v}:=c_{1}\left(\overline{\mathcal{L}}_{v}\right)^{\operatorname{dim} Y} \cdot \delta_{Y_{v}^{\text {an }}} / \operatorname{deg}_{\mathcal{L}}(X) .
$$

One can show an analogue of Proposition 3.1.5, such the support of the measure is any $Z_{v}^{\text {an }}$ for any subvariety $Z$ of $Y$.

We want to propose a generalization of the equidistribution conjecture:

ConjeCture 4.2.2 ( $v$-adic Equidistribution of dynamically generic small points). Let $\phi: X \longrightarrow X$ be an endomorphism of a projective variety over a number field $K$ with a polarization by an ample line bundle $\mathcal{L}$. Let $v$ be a place of $K$. Fix an embedding $v: K_{v} \longrightarrow \mathbb{C}_{p}$ and write $X_{v}^{\text {an }}$ for the induced analytic space. Let $x_{i}$ be a sequence of points on $X$ such that no infinite subsequence is included in a proper preperiodic subvariety, and that $\lim _{i \rightarrow \infty} \widehat{h}_{\mathcal{L}}\left(x_{i}\right)=0$. Then the Galois orbits of $x_{i}$ are equidistributed with respect to the canonical measure on $X_{v}^{\text {an }}$.

REMARK 4.2.3. We would like to consider an adelic version of the above equidistribution. Let $\phi: X \longrightarrow X$ be an endomorphism of a projective variety over a number field $K$ with a polarization by an ample line bundle $\mathcal{L}$. Let $S$ be a finite set of places $K$. For each place $v \in S$, fix an embedding $v: K_{v} \longrightarrow \mathbb{C}_{p}$ and write $X_{v}^{\text {an }}$ for the induced analytic space. Let $d \mu_{v}$ denote the probability measure on $X_{v}^{\text {an }}$. Let $x_{i}$ be a sequence of points on $X$ such that no infinite subsequences are included in a proper preperiodic subvariety, and that $\lim _{i \rightarrow \infty} \widehat{h}_{\mathcal{L}}\left(x_{i}\right)=0$. Then we want to conjecture that the Galois orbits of $x_{i}$ are equidistributed with respect to the canonical measure on $\prod X_{v}^{\text {an }}$, with respect to the product measures $d \mu_{v}$. 
In one special case where $S$ is the set of places over a prime $p$, we may reduce the conjecture for the dynamical system on $\operatorname{Res}_{K / \mathbb{Q}}(X)$ at the place $p$. Indeed, by definition $\operatorname{Res}_{K / \mathbb{Q}}(X)(L)=X(K \otimes L)$. Thus its fiber over $p$ is given by $X \otimes_{K}\left(K \otimes \mathbb{Q}_{p}\right)=\prod_{v \mid p} X_{v}$.

REMARK 4.2.4. We still have some topological interpretation of the above conjecture by introducing the distributional topology on $X_{v}^{\text {an }}$. We will still have a conjecture that the support of $d \mu_{v}$ is Zariski dense. Also we have the analogue of Proposition 4.1.2: the $v$-adic equidistribution conjecture plus the density of the support of $\left(d \mu_{v}\right)$ will imply the "if" part of Conjecture 1.2.1.

Remark 4.2.5. In [50], Szpiro and Tucker gave a formula for canonical heights for the dynamical system on $\mathbb{P}^{1}$ by working on successive blow-ups.

4.3. A generic equidistribution theorem. In this section we want to show that the Bogomolov conjecture is equivalent to the equidistribution conjecture. This is actually a consequence of the following equidistribution theorem for Galois orbits of generic sequences of small points:

THEOREM 4.3.1 (Equidisdibution for Zariski generic small points [57], [64], [16], [60]). Let $X$ be a projective variety over a number field $K$ of dimension $n$. Let $v$ be a place of $K$. Let $\overline{\mathcal{L}}=\left(\mathcal{L},\|\cdot\|_{v}\right)$ be a metrized line bundle on $X$ such that the following hold:

(1) $\mathcal{L}$ is ample,

(2) $\|\cdot\|_{v}$ is semipositive,

(3) $h_{\overline{\mathcal{L}}}(X)=0$.

Let $x_{n}$ be a sequence of points on $X$ such that $\lim h_{\overline{\mathcal{L}}}\left(x_{n}\right)=0$ and that no infinite subsequence of $x_{n}$ is included in a proper subvariety. Then the Galois orbits of $x_{n}$ are equidistributed in $X_{v}^{\text {an }}$ with respect to the measure

$$
d \mu:=c_{1}\left(\mathcal{L}_{v},\|\cdot\|_{v}\right)^{n} / \operatorname{deg}_{\mathcal{L}}(X) .
$$

Corollary 4.3.2. The dynamic Bogomolov Conjecture 4.1 .7 is equivalent to the equidistribution conjecture 4.1 .8 and 4.2.2.

Proof. By a standard trick, we need only show that any infinite subsequence $x_{i_{n}}$ contains another infinite subsequence whose Galois orbits are equidistributed. With $x_{i_{n}}$ replaced by a subsequence we may assume the following:

(1) the Zariski closure $Y$ of $\left\{\Gamma x_{i_{n}}, n=1, \ldots\right\}$ is an integral subvariety of $X$;

(2) no infinite subsequence of $x_{i_{n}}$ is included into a proper subvariety of $Y$.

By the Bogomolov conjecture, $Y$ is a preperiodic subvariety of $X$. By the assumption of the conjecture 4.2.2, $X=Y$. Now Theorem 4.3.1 gives the equidistribution of Galois orbits. 
Sketch of proof of Theorem 4.3.1. The theorem was first proved in SzpiroUllmo-Zhang [57] when $X_{K}$ is smooth and the curvature of $\overline{\mathcal{L}}$ is smooth and positive point-wise on $X(\mathbb{C})$, and extended in $[64]$ when $X_{\mathbb{C}}$ is a subvariety of a smooth variety $Y$ and $\overline{\mathcal{L}}_{\mathbb{C}}$ is the restriction of a metrized line bundle $\mathcal{M}$ with smooth and positive curvature point-wise. Then Chambert-Loir [16] further extended all of these results to $v$-adic Berkovich spaces. The general case stated here is due to Yuan [60] as a consequence of his theorem of arithmetic bigness of line bundles:

TheOREM 4.3.3 (Yuan [60]). Let $\overline{\mathcal{L}}_{1}$ and $\overline{\mathcal{L}}_{2}$ be two arithmetically ample line bundles on $X$ such that

$$
c_{1}\left(\overline{\mathcal{L}}_{1}\right)^{n+1}-(n+1) c_{1}\left(\overline{\mathcal{L}}_{1}\right)^{n} \cdot c_{1}\left(\overline{\mathcal{L}}_{2}\right)>0 .
$$

Then the bundle $\overline{\mathcal{L}}_{1} \otimes \overline{\mathcal{L}}_{2}^{-1}$ is big in the following sense:

$$
\log \#\left\{s \in \Gamma\left(X,\left(\mathcal{L}_{1} \otimes \mathcal{L}_{2}^{-1}\right)^{k}\right):\|s\|_{\text {sup }} \leq 1\right\} \geq c k^{n+1}+o\left(k^{n+1}\right)
$$

where $\|\cdot\|_{\sup }=\sup _{v}\|\cdot\|_{v \text {,sup }}$ is the superum norm over all places and $c$ is a positive number independent of $k$.

Let $f$ be a semi-smooth function on $X_{v}^{\text {an }}$. For each $t \in \mathbb{R}$, let $\overline{\mathcal{L}}_{t}$ denote the metrized line bundle $\left(\mathcal{L},\|\cdot\|_{t}\right)$ with adelic metric $\|\cdot\|_{t}=\|\cdot\| e^{-f t}$ (which differs from $\|\cdot\|$ only at $v)$. Since $f$ is smooth, we have a line bundle $\mathcal{O}_{X}(f)$ on a model of $X$ induces the metric on $\mathcal{O}_{X}$ such that the induced metric has property $\|1\|_{v}=e^{f}$ and $\|1\|_{w}=1$ for $w \neq v$. It is easy to see that there are two semi-ample line bundles $\overline{\mathcal{M}}_{1}$ and $\overline{\mathcal{M}}_{1}$ such that

$$
\mathcal{O}(f)=\overline{\mathcal{M}}_{1} \otimes \overline{\mathcal{M}}_{2}^{-1} \text {. }
$$

Now we have the expression

$$
c_{1}\left(\overline{\mathcal{L}}_{t}\right)=c_{1}\left(\overline{\mathcal{L}} \otimes \overline{\mathcal{M}}_{1}^{t}\right)-c_{1}\left(\overline{\mathcal{M}}_{2}^{t}\right)
$$

Now we apply Yuan's bigness theorem to the two line bundles on the right hand side. The quantity in Yuan's theorem is

$$
\begin{aligned}
& \left(c_{1}(\overline{\mathcal{L}})+t c_{1}\left(\overline{\mathcal{M}}_{1}\right)\right)^{n+1}-(n+1)\left(c_{1}(\overline{\mathcal{L}})+t c_{1}(\overline{\mathcal{M}})\right)^{n}\left(t \bar{c}_{1}\left(\mathcal{M}_{2}\right)\right) \\
& =t c_{1}(\overline{\mathcal{L}})^{n}\left(c_{1}\left(\overline{\mathcal{M}}_{1}\right)-c_{1}\left(\overline{\mathcal{M}}_{2}\right)\right)+O\left(t^{2}\right) \\
& =(n+1) t c_{1}(\overline{\mathcal{L}})^{n} \cdot c_{1}(\mathcal{O}(f))+O\left(t^{2}\right) \\
& =(n+1) t \int f c_{1}(\overline{\mathcal{L}})^{n}+O\left(t^{2}\right) .
\end{aligned}
$$

Thus $\int f c_{1}(\overline{\mathcal{L}})^{n}>0$ will imply that $\overline{\mathcal{L}}_{t}$ is big. In this case there is a section $s$ of $\overline{\mathcal{L}}_{t}^{k}$ with norm $\leq 1$. Now let us use this section to compute the heights of $x \in X(\bar{K})$ when $x$ not in the divisor of $s$,

$$
h_{\overline{\mathcal{L}}_{t}}(x)=-\frac{1}{k} \frac{1}{\operatorname{deg} x} \sum_{v} \sum_{\sigma: K(x) \longrightarrow \bar{K}_{v}} \log \|s\|_{v}(\sigma(x)) \geq 0 .
$$


On the other hand, we can compute the height by the additivity

$$
\overline{\mathcal{L}}_{t}=\overline{\mathcal{L}}+O(t f)
$$

and Section 1 for $O(t f)$ with norm $e^{-t f}$ :

$$
\begin{aligned}
h_{\overline{\mathcal{L}}_{t}}(x) & =h_{\overline{\mathcal{L}}}(x)+h_{\mathcal{O}(t f)}(x) \\
& =h_{\overline{\mathcal{L}}}(x)+\frac{t}{\operatorname{deg} x} \sum_{\sigma: K(x) \longrightarrow \bar{K}_{v}} f(\sigma(x)) .
\end{aligned}
$$

Combining both expressions we obtain that $\int f c_{1}(\overline{\mathcal{L}})^{n}>0$ implies that for $x$ in a Zariski dense subset,

$$
\frac{t}{\operatorname{deg} x} \sum_{\sigma: K(x) \longrightarrow \bar{K}_{v}} f(\sigma(x)) \geq-h_{\overline{\mathcal{L}}}(x) .
$$

Now we apply this inequality to $x_{n}$ in Theorem 4.3 .1 to obtain that

$$
\lim \inf _{n \longrightarrow \infty} \frac{t}{\operatorname{deg} x_{n}} \sum_{\sigma: K\left(x_{n}\right) \longrightarrow \bar{K}_{v}} f\left(\sigma\left(x_{n}\right)\right) \geq 0 .
$$

For arbitrary $f$, we may replace $f$ by $f-\int f d \mu+\epsilon$ (which has positive integral $\epsilon>0$ ) to obtain the following unconditional inequality

$$
\lim \inf _{n \longrightarrow \infty} \frac{1}{\operatorname{deg} x} \sum_{\sigma: K\left(x_{n}\right) \longrightarrow \bar{K}_{v}} f\left(v\left(x_{n}\right)\right) \geq \int f d \mu .
$$

We may replace $f$ by $-f$ in the above expression to obtain

$$
\lim \sup _{n \longrightarrow \infty} \frac{1}{\operatorname{deg} x} \sum_{\sigma: K\left(x_{n}\right) \longrightarrow \bar{K}_{v}} f\left(\sigma\left(x_{n}\right)\right) \leq \int f d \mu .
$$

Thus we have shown that

$$
\lim _{n \longrightarrow \infty} \frac{1}{\operatorname{deg} x} \sum_{\sigma: K\left(x_{n}\right) \longrightarrow \bar{K}_{v}} f\left(\sigma\left(x_{n}\right)\right)=\int f d \mu .
$$

REMARK 4.3.4. When $\operatorname{dim} X=1$, Theorem 4.3.1 has been proved by A. Chambert-Loir in [17] using a bigness type result of Autissier [1] for arithmetic surfaces. In the spacial case where $X=\mathbb{P}^{1}$, and $\mathcal{L}$ is equipped with the canonical metric induced from an endomorphism $\phi$ of degree $>1$, Theorem 4.3.1 has been proved by two different groups of people: M. Baker and R. Rumely [3] and C. Favre and J. Rivera-Letelier in [24]. 


\section{References}

[1] P. Autissier, Points entiers sur les surfaces arithmétiques, J. Reine Angew. Math. 531 (2001).

[2] P. Autissier, Dynamique des correspondances algebriques et hauteurs, Int. Math. Res. Not. 69 (2004).

[3] M. Baker and R. Rumely, Equidistribution of small points, rational dynamics, and potential theory, arXiv: math.NT/0407426.

[4] D. Barlet, Espace analytique réduit des cycles analytiques complexes compacts d'un espace analytique complexe de dimension finie, in 'Fonctions de plusieurs variables complexes, II', Lecture Notes in Math., 482, 1-158. Springer, Berlin, 1975.

[5] A. Beauville, Variétés kähleriennes dont la première classe de Chern est nulle, Journal of Differential Geometry 87 (1983).

[6] A. Beauville, Endomorphisms of hypersurfaces and other manifolds, Internat. Math. Res. Notices 1 (2001), 53-58.

[7] V.G. Berkovich, Spectral theory and analytic geometry over non-Archimedean fields, Mathematical Surveys and Monographs, 33, American Mathematical Society, Providence, RI, 1990.

[8] Y. Bilu, Limit distribution of small points on algebraic tori, Duke Mathematical Journal 89(3) (1997).

[9] J.-V. Briend and J. Duval, Deux caractérisations de la mesure d'équilibre d'un endomorphisme de $\mathbf{P}^{k}(\mathbf{C})$, Publ. Math. Inst. Hautes Études Sci. 93 (2001), 145-159.

[10] J.-Y. Briend, S. Cantat, and M. Shishikura, Linearity of the exceptional set for maps of $\mathbb{P}^{k}(\mathbb{C})$, Math. Ann. 330(1) (2004), 39-43.

[11] G. Call and J.H. Silverman, Canonical heights on varieties with morphisms, Compositio Math. 89(2) (1993), 163-205.

[12] F. Campana, Connexité rationnelle des variétés de Fano, Ann. Sci. école Norm. Sup. (4) 25 (1992), 539-545.

[13] F. Campana, Orbifolds, special varieties and classification theory, Ann. Inst. Fourier (Grenoble) 54(3) (2004), 499-630.

[14] S. Cantat, Dynamique des automorphismes des surfaces K3, Acta Math. 187(1), $1-57$.

[15] S. Cantat, Dynamique des automorphismes des surfaces projectives complexes, C.R. Acad. Sci. Paris Sér. I Math. 328(10) (1999), 901-906.

[16] A. Chambert-Loir, Mesures et équidistribution sur les espaces de Berkovich, arXiv: math.NT/0304023 v3, 12 Nov 2004.

[17] A. Chambert-Loir, Points de petite hauteur sur les variétés semi-abéliennes, Ann. Sci. école Norm. Sup. (4) 33(6) (2000), 789-821.

[18] S. David and P. Philippon, Minorations des hauteurs normalisées des sous-variétés de variétés abéliennes, in 'Number theory', Contemp. Math., 210, Amer. Math. Soc., 1998.

[19] P Deligne, Le déterminant de la cohomologie, in 'Current trends in arithmetical algebraic geometry' (Arcata, Calif., 1985), Contemp. Math., 67, 93-177, Amer. Math. Soc., Providence, RI, 1987.

[20] J.-P. Demailly, Mesures de monge-ampére et caractérisation géométrique des variétés algébriques affines, Mém. Soc. Math. France (N.S.), 1-125, 1985.

[21] T. Dinh and N. Sibony, Dynamique des endomorphismes holomorphes, preprint, 2002.

[22] T. Dinh and N. Sibony, Groupes commutatifs d'automorphismes d'une variété kählérienne compacte, Duke Math. J. 123(2) (2004), 311-328.

[23] N. Fakhruddin, Questions on self maps of algebraic varieties, J. Ramanujan Math. Soc. 18(2) (2003), 109-122.

[24] C. Favre and J. Rivera-Letelier, Equidistribution quantitative des points de petite hauteur sur la droite projective, arXiv:math. NT/0407471. 
[25] Y. Fujimoto and N. Nakayama, Compact complex surfaces admitting non-trivial surjective endomorphisms, Tohoku Mathematical Journal 57(3) (2005), 395-426.

[26] H. Gillet and C. Soulé, Arithmetic intersection theory, Inst. Hautes Etudes Sci. Publ. Math. 72 (1991).

[27] P. Griffiths and J. Harris, Principles of algebraic geometry, Wiley-Interscience, 1978.

[28] P. Griffiths, Entire holomorphic mappings in one and several complex variables. Princeton University Press, 1976.

[29] W. Gubler. Höhentheorie, with an appendix by jurg kramer, Math. Ann. 298(3) (1994), 427-455.

[30] W. Gubler, Local heights of subvarieties over non-archimedean fields, J. Reine Angew. Math. 498 (1998), 61-113.

[31] J. Harris and Y. Tschinkel, Rational points on quartics, Duke Math. J. 104(3) (2000), 477-500.

[32] A. Katok and B. Hasselblatt, Introduction to the Mordern Theory of Dynamical Systems, Cambridge University Press, 1995.

[33] S. Kawaguchi, Canonical heights, invariant currents, and dynamical systems of morphoisms associated to line bundles, math.NT/0405006.

[34] S. Kobayashi, Intrinsic distances, measures and geometric function theory, Bull. Amer. Math. Soc. 82(3) (1976), 357-416.

[35] J. Kramer, On a generalization of the Neron-Tate height on abelian varieties, in 'Abelian varieties' (Egloffstein, 1993), 199-209, de Gruyter, Berlin, 1995.

[36] S. Lang, Fundamentals of Diophantine geometry, Springer-Verlag, 1983.

[37] M. Laurent, Équations diophantiennes exponentielles, Inventiones Mathematicae 78(2) (1984), 299-327.

[38] B. Mazur, The topology of rational points, J. EXp. Math. 1 (1992), 35-46.

[39] C. McMullen, Dynamics on blowups of the projective plane.

[40] C. McMullen, Dynamics on K3 surfaces: Salem numbers and siegel disks, J. reine angen. Math. 545 (2002), 201-233.

[41] J. Milnor, Dynamics in one complex variable, Annals of Mathematics Studies, 160, Princeton University Press, Princeton, NJ, 3 edition, 2006.

[42] A. Mimar, PhD thesis, 1995.

[43] Y. Miyaoka and S. Mori, A numerical criterion for uniruledness, Ann. of Math. 124(1) (1986), 65-69.

[44] S. Mori, Flip theorem and the existence of minimal models for 3-folds, J. Amer. Math. Soc. 1(1) (1988), 117-253.

[45] P. Morton and J.H. Silverman, Rational periodic points of rational functions, Internat. Math. Res. Notices.

[46] N. Nakayama, Ruled surfaces with non-trivial surjective endomorphism, Kyushu Journal of Mathematics (2), 2002.

[47] D.G. Northcott, Periodic points on an algebraic variety, Ann. of Math. (2) 51 (1950), 167-177.

[48] T. Peternell, Towards a Mori theory on compact Kähler threefolds III, Bull. Soc. Math. France 129(3) (2001), 339-356.

[49] P. Philippon, Sur des hauteurs alternatives, I, Math. Ann. 289(2) (1991), 255-283.

[50] J. Pineiro, L. Szpiro, and T. Tucker. Mahler measure for dynamical systems on $\mathbb{P}^{1}$ and intersection theory on a singular arithmetic surface, Geometric Methods in Algebra and Number Theory, 235, 2004.

[51] M. Paun, S. Bouchson, J.-P. Demailly and T. Peternell, The pseudo-effective cone of a compact Kähler manifold and varieties of negative kodaira dimension, arXiv:math.AG/0405285 v1, 14 May 2004.

[52] M. Raynaud, Sous-variétés d'une variété abélienne et points de torsion, in 'Arithmetic and Geometry' (J. Coates and S. Helgason, editors), 1, Birkauser, 1983. 
[53] J.-P. Serre, Analogues kählériens de certaines conjectures de Weil, Annales of Mathematics 71 (1960), 392-394.

[54] N. Sibony, Dynamique des applications rationnelles de $\mathbb{P}^{k}$, in 'Dynamique et géométrie complexes', Panor. Synthéses, 8, 97-185, Soc. Math. France, Paris, 1999.

[55] J.H. Silverman, Rational points on K3 surfaces: a new canonical height, Invent. Math. 105(2) (1991), 347-373.

[56] J.H. Silverman, The space of rational maps on $\mathbb{P}^{1}$, Duke Math. J. 94(1) (1998), 41-77.

[57] L. Szpiro, E. Ullmo, and S. Zhang, Équirépartition des petits points, Invent. Math. 127(2) (1997).

[58] E. Ullmo, Positivité et discrétion des points algébriques des courbes, Ann. of Math. (2) $\mathbf{1 4 7}(\mathbf{1})$ (1998).

[59] S.-T. Yau, On the Ricci curvature of a compact Käler manifold and the complex Monge-Ampere equation, I, Comm. Pure Appl. Math. 31(3) (1978), 339-411.

[60] X. Yuan, Ph.D. thesis, in preparation.

[61] S.-W. Zhang, Positive line bundles on arithmetic varieties, J. Amer. Math. Soc. 8(1) (1995).

[62] S.-W. Zhang, Small points and adelic metrics, Journal of Algebraic Geometry 4(2) (1995).

[63] S.-W. Zhang, Heights and reductions of semi-stable varieties, Compositio Math. 104(1) (1996), 77-105.

[64] S.-W. Zhang, Equidistribution of small points on abelian varieties, Ann. of Math. (2) 147(1) (1998).

Columbia University, 2990 Broadway, 509 Mathematics Buidling,

NY, NY 10027

E-mail address: szhang@cpw.math.columbia.edu 